\title{
Characterization of Novel Fluorescent Bile Salt Derivatives for Studying Human Bile Salt and Organic Anion Transporters ${ }^{[\mathrm{S}}$
}

\author{
Michele Leuenberger, ${ }^{1}$ Stephanie Häusler, ${ }^{1}$ Vera Höhn, Adriana Euler, Bruno Stieger, \\ and (1) Martin Lochner \\ Institute of Biochemistry and Molecular Medicine, University of Bern, Bern, Switzerland (M.Le., M.Lo.); Department of Clinical \\ Pharmacology and Toxicology, University Hospital Zürich, Zürich, Switzerland (S.H., V.H., A.E., B.S.); and Swiss National Center \\ of Competence in Research, NCCR TransCure, Bern, Switzerland (M.Le., S.H., A.E., B.S., M.Lo.)
}

Received December 2, 2020; accepted March 23, 2021

\begin{abstract}
Bile salts, such as cholate, glycocholate, taurocholate, and glycochenodeoxycholate, are taken up from the portal blood into hepatocytes via transporters, such as the $\mathrm{Na}^{+}$-taurocholatecotransporting polypeptide (NTCP) and organic anion-transporting polypeptides (OATPs). These bile salts are later secreted into bile across the canalicular membrane, which is facilitated by the bile salt export pump (BSEP). Apart from bile salt transport, some of these proteins (e.g., OATPs) are also key transporters for drug uptake into hepatocytes. In vivo studies of transporter function in patients by using tracer compounds have emerged as an important diagnostic tool to complement classic liver parameter measurements by determining dynamic liver function both for diagnosis and monitoring progression or improvement of liver diseases. Such approaches include use of radioactively labeled bile salts (e.g., for positron emission tomography) and fluorescent bile salt derivatives or dyes (e.g., indocyanine green). To expand the list of liver function markers, we synthesized fluorescent derivatives of cholic and chenodeoxycholic acid by conjugating small organic dyes to the bile acid side chain. These novel fluorescent probes were able to block substrate transport in a concentration-dependent manner of NTCP, OATP1B1,
\end{abstract}

OATP1B3, OATP2B1, BSEP, and intestinal apical sodiumdependent bile salt transporter (ASBT). Whereas the fluorescent bile acid derivatives themselves were transported across the membrane by OATP1B1, OATP1B3, and OATP2B1, they were not transport substrates for NTCP, ASBT, BSEP, and multidrug resistance-related protein 2. Accordingly, these novel fluorescent bile acid probes can potentially be used as imaging agents to monitor the function of OATPs.

\section{SIGNIFICANCE STATEMENT}

Synthetic modification of common bile acids by attachment of small organic fluorescent dyes to the bile acid side chain resulted in bright, fluorescent probes that interact with hepatic and intestinal organic anion [organic anion-transporting polypeptide (OATP) 1B1, OATP1B3, OATP2B1], bile salt uptake $\left(\mathrm{Na}^{+}\right.$-taurocholate-cotransporting polypeptide, apical sodiumdependent bile salt transporter), and bile salt efflux (bile salt export pump, multidrug resistance-related protein 2) transporters. Although the fluorescent bile salt derivatives are taken up into cells via the OATPs, the efflux transporters do not transport any of them but one.

\section{Introduction}

Bile formation is one of several important liver functions (Stieger, 2011). The liver is also a second barrier for preventing

This work was supported by the Swiss National Science Foundation through the National Center of Competence in Research (NCCR) TransCure [Grant 51NF40-160620] and the University of Bern.

No author has an actual or perceived conflict of interest or financial conflict with the contents of this article. Chemical structures of all new compounds cited or discussed in the manuscript are presented, or a citation to the published literature is provided

${ }^{1}$ M.Le. and S.H. contributed equally to the work.

https://doi.org/10.1124/jpet.120.000449.

S This article has supplemental material available at jpet.aspetjournals.org. the entry of harmful substances into the body via the oral route because of its role in detoxification (Corsini and Bortolini, 2013; Selvapatt et al., 2014). Consequently, the liver is constantly at risk of insults by xenobiotics, which may lead to liver injury in susceptible patients (Corsini and Bortolini, 2013).

The major constituents of bile are bile salts (BSs), which are organic anions like conjugates of bilirubin and lipids (Esteller, 2008). BSs are absorbed from the portal blood into hepatocytes from which they are secreted across the canalicular membrane into bile draining into the small intestine. In the intestine, BSs are almost quantitatively absorbed and transported back into the liver by the portal vein, where their journey starts again. The transport systems involved in hepatocellular handling of

ABBREVIATIONS: abs, absorption; Acen, acetyl ethylenediamine; ASBT, apical sodium-dependent bile salt transporter; BA, bile acid; br, broad peak; BS, bile salt; BSEP, bile salt export pump; CDCen, (2-aminoethyl)chenodeoxycholic amide; Cen, (2-aminoethyl)cholic amide; d, doublet; DCM, dichloromethane; DMF, N,N-dimethylformamide; DNS, Dansyl, 5-(dimethylamino)naphthalene-1-sulfonamide; em, emission; EtOAc, ethyl acetate; ex, excitation; eq., equivalent; FCS, fetal calf serum; Fluo, fluorescence; HRMS, high-resolution mass spectra; LC, liquid chromatography; $\mathrm{m}$, multipletMeOH methanol $\mathrm{m} / \mathrm{z}$ mass-to-charge ratio; MRP, multidrug resistance-related protein; MS, mass spectrometry; NBD, 4-nitrobenzo[c][1,2,5]oxadiazole; NTCP, Na+-taurocholate-cotransporting polypeptide; OATP, organic anion-transporting polypeptide; OST, organic solute transporter; PB, Pacific Blue, 6,8-difluoro-7-hydroxy-2-oxo-2H-chromene-3-carboxamide; q, quartet; s, singlet; t, triplet; TLC, thinlayer chromatography; WT, wild type. 
bile acids (BAs) and BSs are known (Stieger, 2011; Pollheimer et al., 2014) and include for the uptake from the blood plasma the $\mathrm{Na}^{+}$-taurocholate-cotransporting polypeptide (NTCP) and the organic anion-transporting polypeptides (OATPs) OATP1B1, OATP1B3, and OATP2B1. Efflux of BS from hepatocytes into bile is mediated by the BS export pump (BSEP). In the intestine, BSs are reclaimed by the apical sodium-dependent BS transporter (ASBT) in the apical membrane of enterocytes in the distal ileum. Export from enterocytes is accomplished through the basolateral membrane by the organic solute transporter (OST) $\alpha / \mathrm{OST} \beta$ (Dawson and Karpen, 2015). In hepatocytes, MRP3, MRP4, and OST $\alpha / \mathrm{OST} \beta$ are expressed at the basolateral membrane and may act as salvage transporters for BS in cholestatic conditions (Halilbasic et al., 2013).

BS export by BSEP constitutes the rate-limiting step of hepatocellular BS flux. Inherited or acquired impairment of BSEP function may lead to liver disease (Aithal et al., 2011; Bull and Thompson, 2018). The substrate specificity of BSEP is restricted with very few exceptions to BS (Stieger, 2011). Similarly, the main substrates for NTCP and ASBT are BSs (Anwer and Stieger, 2014). In addition to contributing to BA uptake into hepatocytes, OATPs have a broad substrate specificity and are key transporters for drug uptake (Hagenbuch and Stieger, 2013).

Measurements of so-called liver parameters are an important part of diagnosing liver disease. These parameters often only allow indirect conclusions on liver function. For example, serum-bilirubin levels give indirect information on the uptake, metabolic, and excretory/secretory functions of the liver (Hoekstra et al., 2013). To obtain more direct functional (or dynamic) information on the liver, in vivo determination of hepatocellular transporter function has become an active area of translational research (Stieger et al., 2012). Recent data show, for instance, that in vivo determination of liver function by monitoring hepatobiliary transport of ${ }^{99 \mathrm{~m}} \mathrm{Tc}$-mebrofenin with scintigraphy improves the outcome of risk assessment for patients needing major liver resection (Cieslak et al., 2016).

It was recognized that BSs are elevated in plasma in patients with liver disease (Frosch and Wagener, 1967), and it was later demonstrated that in such patients the clearance of glycocholate is reduced (LaRusso et al., 1975) and that the clearances of taurocholate and indocyanine green correlate well (Paumgartner et al., 1979). However, because of the endogenous BS pool, clearance measurements require radioactive BS (LaRusso et al., 1975). BS transport in vivo is currently explored by positron emission tomography, wherein hepatic transport of a BA derivative can be monitored in healthy individuals and patients (Cieslak et al., 2016; Kjærgaard et al., 2021). Such techniques expose patients to internal radiation and require complex methodologies. An alternate method is using fluorescence measurements. Spectrophotometric determination of indocyanine green clearance is routinely used to assess liver function (Stieger et al., 2012) and can also be performed transcutaneously, allowing continuous monitoring of liver function (Sakka, 2018). A pilot study demonstrated that the plasma clearance of the fluorescent BS cholyl-lysyl-fluorescein is reduced in patients with liver cirrhosis compared with healthy controls (Milkiewicz et al., 2000). However, this BS derivative is neither a substrate of NTCP nor BSEP (de Waart et al., 2010). Other fluorescein-BS conjugates like CGamF (Holzinger et al., 1997, 1998) and, more recently, ${ }^{18} \mathrm{~F}$-labeled BS derivatives (Testa et al., 2017) have been studied as potential tracers of hepatic transporters in rat.

In this work, we aimed to test novel fluorescent BAs as potential liver function indicators. We synthesized novel fluorescent BA conjugates by modifying the side chain and tested their interaction with BS transporters using in vitro test systems. Although the new fluorescent BS conjugates inhibited all tested transporters except MRP2, these conjugates were substrates to OATPs only, albeit to different extents.

\section{Materials and Methods}

\section{Materials Transporter Biology}

Radiolabeled $\left[6,7-{ }^{3} \mathrm{H}(\mathrm{N})\right]$-estradiol $17 \beta$-D-glucuronide, $\left[6,7-{ }^{3} \mathrm{H}(\mathrm{N})\right]$ estrone sulfate, $\left[{ }^{3} \mathrm{H}(\mathrm{G})\right]$-taurocholic acid, and scintillation cocktail Ultima Gold were obtained from PerkinElmer (Schwerzenbach, Switzerland). [6,7- $\left.{ }^{3} \mathrm{H}(\mathrm{N})\right]$-estradiol $17 \beta$-D-glucuronide and taurocholic acid sodium salt were from Sigma-Aldrich (St. Louis, MO). Cell Culture Material: CHO-Flp-In cells and LipofectAMINE 2000 were from Invitrogen (Carlsbad, CA). The baculovirus-expressing MRP2 (de Waart et al., 2010) was the generous gift of Drs. Paulusma and Oude Elferinik, Academic Medical Center, Amsterdam (The Netherlands).

\section{Transporter Expression Systems}

CHO cells stably expressing OATP1B1, OATP1B3, or OATP2B1 were characterized by Treiber et al. (2007) and Gui et al. (2008). The cells were maintained in Dulbecco's modified Eagle's medium supplemented with $10 \%$ (v/v) fetal calf serum (FCS): $50 \mu \mathrm{g} / \mathrm{ml}$ L-proline, 100 $\mathrm{U} / 100 \mu \mathrm{g}$ per $\mathrm{ml}$ penicillin/streptomycin, and $500 \mu \mathrm{g} / \mathrm{ml}$ geneticin sulfate for transfected cells.

The generation of CHO Flp-In cells stably expressing NTCP was reported by de Waart et al. (2010). The CHO cell line stably expressing ASBT was established as follows: CHO Flp-In cells were cotransfected with pcDNATM5/FRT-hASBT (Zhao et al., 2015) and pOG44 (ratio 1:9) using the LipofectAMINE 2000 method according to the manufacturer's protocol. Cells were selected in medium supplemented with $500 \mu \mathrm{g} / \mathrm{ml}$ hygromycin B, and single clones were obtained by the use of cloning cylinders. The clones were assessed by determination of mRNA expression levels and taurocholate transport activity. CHO Flp-In cells were cultured in HAM-F12 medium enriched with $10 \%$ (v/v) FCS, $1 \mathrm{mM}$ L-glutamine, $100 \mathrm{U} / 100 \mu \mathrm{g}$ per $\mathrm{ml}$ penicillin/streptomycin, $100 \mu \mathrm{g} / \mathrm{ml}$ Zeocin (control cells), or $500 \mu \mathrm{g} / \mathrm{ml}$ hygromycin B (transfected cells).

The efflux transporter BSEP (Noé et al., 2002) and MRP2 (de Waart et al., 2006) were expressed with the baculovirus system in Sf21 cells, and membrane vesicles were isolated as described (Gerloff et al., 1998). Sf21 cells were grown Grace's insect medium containing $10 \%(\mathrm{v} / \mathrm{v})$ heat-inactivated FCS and penicillin/streptomycin at $100 \mathrm{U} / 100 \mu \mathrm{g}$ per $\mathrm{ml}$.

\section{Transport Experiments}

Cell culture and transport experiments with radioactively labeled substrates were performed as detailed by Wlcek et al. (2013). Prior to transport experiments, the cells were cultured for 24 hours in the respective medium containing $5 \mathrm{mM}$ sodium butyrate to increase protein expression of the transporters (Palermo et al., 1991). The following substrate concentrations were used for transport experiments with radioactively labeled substrates: $1 \mu \mathrm{M}$ taurocholate for ASBT, $5 \mu \mathrm{M}$ taurocholate for NTCP, $1 \mu \mathrm{M}$ estrone-3-sulfate for OATP1B1 and OATP2B1, $5 \mu \mathrm{M}$ taurocholate for OATP1B3, $10 \mu \mathrm{M}$ estradiol $17 \beta$-D-glucuronide for MRP2, and $2 \mu \mathrm{M}$ taurocholate for 
BSEP. For the kinetic characterization of the established ASBT cell line, initial uptake rates were first determined (unpublished data). To test fluorescent compounds as substrates, they were in all experiments used at a concentration of $5 \mu \mathrm{M}$. In case of fluorescently labeled bile salts, the cells were solubilized after the transport experiment for at least 30 minutes with $1 \mathrm{ml}$ of $1 \%$ (w/v) Triton X100 , and fluorescence was determined in a PerkinElmer LS-55 fluorescence spectrometer.

Transport experiments with vesicles isolated from Sf21 cells were described by Wlcek et al. (2013). In case of radioactive substrates, the filters were dissolved in Filter Count liquid scintillation cocktail from PerkinElmer. In case of experiments with fluorescent substrates, after the uptake the filters were incubated in $1 \mathrm{ml}$ of $1 \%(\mathrm{w} / \mathrm{v})$ Triton X-100 in an Eppendorf tube for 30 minutes and subsequently centrifuged for 40 minutes in an Eppendorf FA-45-30-11 rotor at 20,000g. The supernatant was used for fluorescence determination in a Perkin Elmer LS-55 fluorescence spectrometer.

\section{Analytical Methods}

The following settings were used for fluorescence measurements: for Dansyl (DNS) derivatives, excitation (ex) $335 \mathrm{~nm}$ (slit $20 \mathrm{~nm}$ ) and emission (em) $520 \mathrm{~nm}$ (slit $20 \mathrm{~nm}$ ); for 4-nitrobenzo[c][1,2,5] oxadiazole (NBD) derivatives, ex $478 \mathrm{~nm}$ (slit $20 \mathrm{~nm}$ ) and em $545 \mathrm{~nm}$ (slit $20 \mathrm{~nm}$ ); and for Pacific Blue (PB) derivatives, ex $410 \mathrm{~nm}$ (slit $5 \mathrm{~nm}$ ) and em $451 \mathrm{~nm}$ (slit $5 \mathrm{~nm}$ ). Standard curves were prepared with the fluorescent substrates in $1 \%(\mathrm{w} / \mathrm{v})$ Triton X-100. A total membrane fraction of WT and ASBT-expressing cells was isolated as described by Huber et al. (2007) and used for Western blotting (Mahdi et al., 2016). Protein was quantified with the bicinchoninic acid method (Smith et al., 1985) using the BCA protein assay kit from Interchim (Montluçon, France).

\section{Chemistry General Remarks}

Reagents and solvents were purchased from commercial sources and were used without further purification or drying. Automated column chromatography was performed on a TeledyneISCO CombiFlash Rf + machine on RediSepRf prepacked columns. Progress of chemical reactions was monitored on Alugram Xtra Sil G/UV254 TLC plates with detection of the compounds by UV-visible absorption at $254 \mathrm{~nm}$ and $366 \mathrm{~nm}$. Compounds were analyzed by UPLC-MS, and their purity was assessed by analytical reversed-phase ultra performance liquid chromatography (RP-UPLC). RP-UPLC spectra were obtained from a Dionex Ultimate 3000 RSLC System and a Dionex Acclaim RSLC 120 column. The following elution method was used: A gradient from $100 \%$ A (milliQ deionized water with $0.05 \%$ trifluoroacetic acid) to $100 \% \mathrm{D}$ (high-pressure liquid chromatography-grade acetonitrile/milliQ deionized water 9:1 with $0.05 \%$ trifluoroacetic acid) was applied for 4.5 minutes, and this was followed by $100 \% \mathrm{D}$ washing. Low-resolution mass spectra were acquired on a Thermo Scientific LCQ Fleet by electron spray ionization in the positive mode. NMR spectra were recorded on a BRUKER Advance $300\left({ }^{1} \mathrm{H}\right.$ at $300 \mathrm{MHz} ;{ }^{13} \mathrm{C}$ at $75 \mathrm{MHz} ;{ }^{19} \mathrm{~F}$ at $282 \mathrm{MHz}$ spectra) and a BRUKER Advance II 400 $\left({ }^{1} \mathrm{H}\right.$ at $400 \mathrm{MHz}$ and ${ }^{13} \mathrm{C}$ at $101 \mathrm{MHz}$ spectra). Chemical shifts are given in parts per million $(\delta)$ referenced to TMS $\left(\delta=0.00 \mathrm{ppm}{ }^{1} \mathrm{H}\right.$ and ${ }^{13} \mathrm{C}$ NMR). The order of citation in parentheses is (1) multiplicity: $\mathrm{s}$ (singlet), d (doublet), t (triplet), q (quartet), m (multiplet), br (broad peak); (2) coupling constant $(J)$ in $\mathrm{Hz}$; and (3) number of equivalent nuclei (by integration). HSQC and HMBC were routinely used to assign peaks in ${ }^{1} \mathrm{H}$ and ${ }^{13} \mathrm{C}$ NMR spectra. High-resolution mass spectra (HRMS) measurements were performed by the Mass Spectrometry Service at the Department of Chemistry and Biochemistry, University of Bern, Switzerland on a Thermo Scientific LTQ OrbitrapXL. Absorption spectra were measured with a Varian Cary 100 Bio UV-visible spectrometer, and emission spectra were measured with a Varian Cary Eclipse fluorescence spectrometer. Copies of ${ }^{1} \mathrm{H},{ }^{13} \mathrm{C}$, and ${ }^{19} \mathrm{~F}$ NMR spectra of synthetic intermediates and final compounds and
UV-Vis absorption and fluorescence emission spectra of fluorescent probes are provided as Supplemental Data.

\section{Compound Synthesis.}

(2-Aminoethyl)cholic Amide. A solution of cholic acid (1 g, $2.45 \mathrm{mmol}, 1$ eq.) in methanol ( $25 \mathrm{ml})$ was treated with acetyl chloride ( $25 \mu \mathrm{l}, 25 \mathrm{mg}, 0.32 \mathrm{mmol}, 0.13$ eq.) at room temperature. The reaction was complete after 45 minutes. The solvent was concentrated in vacuo to give methyl cholate ( $1.1 \mathrm{~g}$, quantitative) as a white foam (containing small amounts of MeOH). ${ }^{1} \mathrm{H}$ NMR (300 MHz, $d_{6}$-DMSO) $\delta 4.02(\mathrm{br} \mathrm{s}$, containing $\mathrm{HDO}, \mathrm{MeOH}$, and the three $\mathrm{OH}), 3.77(\mathrm{~s}, 1 \mathrm{H}), 3.61(\mathrm{~d}, J=$ $3.0,1 \mathrm{H}), 3.57(\mathrm{~s}, 3 \mathrm{H}), 3.27-3.09(\mathrm{~m}, 1 \mathrm{H}$, partially overlapped with the $\mathrm{MeOH}$ signal), 2.44-1.91 (m, $5 \mathrm{H}), 1.88-1.53(\mathrm{~m}, 6 \mathrm{H}), 1.53-0.76(\mathrm{~m}$, 13H). $0.91(\mathrm{~d}, J=6.0,3 \mathrm{H}), 0.80(\mathrm{~s}, 3 \mathrm{H})$, and $0.58(\mathrm{~s}, 3 \mathrm{H})$. HRMS $\mathrm{m} / z$ calculated for $\mathrm{C}_{25} \mathrm{H}_{42} \mathrm{~N}_{5} \mathrm{Na}[\mathrm{M}+\mathrm{Na}]^{+} 445.2924$, which found 445.2917, $\Delta-1.70 \mathrm{ppm}$.

Methyl cholate (1.1 g, $2.62 \mathrm{mmol}, 1$ eq.) was added to ethylenediamine (5 ml, $74.79 \mathrm{mmol}, 29$ eq.), and the resulting solution was heated at reflux $\left(140^{\circ} \mathrm{C}\right)$. After 3 hours the reaction was complete. The reaction solution was then cooled to room temperature, and ice water $(50 \mathrm{ml})$ was added into the solution, after which a white precipitate formed. The solid was filtered, washed with cold water, and dried under reduced pressure to obtain (2-aminoethyl)cholic amide (Cen) (848 mg, 72\%). ${ }^{1} \mathrm{H} \mathrm{NMR} \mathrm{(300} \mathrm{MHz}, d_{6}$-DMSO) $\delta 7.69(\mathrm{t}, J=5.6,1 \mathrm{H})$, $4.30(\mathrm{~s}, 1 \mathrm{H}), 4.09(\mathrm{~s}, 1 \mathrm{H}), 4.00(\mathrm{~d}, J=3.3,1 \mathrm{H}), 3.78(\mathrm{~s}, 1 \mathrm{H}), 3.61(\mathrm{~s}, 1 \mathrm{H})$, $3.18(\mathrm{~s}, 1 \mathrm{H}), 3.00(\mathrm{q}, J=6.2,2 \mathrm{H}), 2.53(\mathrm{q}, J=6.5,2 \mathrm{H}), 2.32-0.74(\mathrm{~m}$, $26 \mathrm{H}), 0.92(\mathrm{~d}, J=6.2,3 \mathrm{H}), 0.81(\mathrm{~s}, 3 \mathrm{H})$, and $0.58(\mathrm{~s}, 3 \mathrm{H})$. HRMS $\mathrm{m} / z$ was calculated for $\mathrm{C}_{26} \mathrm{H}_{47} \mathrm{~N}_{2} \mathrm{O}_{4}[\mathrm{M}+\mathrm{H}]^{+}$451.3530, which found 451.3517, $\Delta-2.98$ ppm. LC-MS $\mathrm{t}_{\mathrm{R}} 2.3$ minutes, $m / z[\mathrm{M}+\mathrm{H}]^{+} 451.28$.

(2-Aminoethyl)chenodeoxycholic Amide. Similarly to the synthesis of Cen above, chenodeoxycholic acid ( $1 \mathrm{~g}, 2.54 \mathrm{mmol}, 1$ eq.) was dissolved in $\mathrm{MeOH}(25 \mathrm{ml})$. Acetyl chloride ( $25 \mu \mathrm{l}, 25 \mathrm{mg}, 0.32 \mathrm{mmol}$, 0.13 eq.) was added at room temperature, and the resulting solution was stirred overnight. The solvent was concentrated in vacuo to give methyl chenodeoxycholate $(1.1 \mathrm{~g}$, quantitative) as a colorless oil. ${ }^{1} \mathrm{H}$ NMR (300 MHz, $d_{6}$-DMSO) $\delta 4.02$ (br s, containing hydrodeoxygenation, $\mathrm{MeOH}$, and the two $\mathrm{OH}), 3.77(\mathrm{~s}, 1 \mathrm{H}), 3.62(\mathrm{~d}, J=$ $2.8,1 \mathrm{H}), 3.57(\mathrm{~s}, 3 \mathrm{H}), 3.27-3.08(\mathrm{~m}, 1 \mathrm{H}$, partially overlapped with the MeOH signal), 2.42-2.08 (m, 3H), 1.97-1.53 (m, 7H), 1.53-0.78 $(\mathrm{m}, 16 \mathrm{H}), 0.88(\mathrm{~d}, J=6.4,3 \mathrm{H}), 0.83(\mathrm{~s}, 3 \mathrm{H}), 0.60(\mathrm{~s}, 3 \mathrm{H})$. HRMS $m / z$ was calculated for $\mathrm{C}_{25} \mathrm{H}_{42} \mathrm{NaO}_{4}[\mathrm{M}+\mathrm{Na}]^{+} 429.2975$, which found 429.2964, $\Delta-2.66 \mathrm{ppm}$.

Methyl chenodeoxycholate ( $1.1 \mathrm{~g}, 2.68 \mathrm{mmol}, 1 \mathrm{eq}$.) was added to ethylenediamine ( $5 \mathrm{ml}, 74.79 \mathrm{mmol}, 28 \mathrm{eq}$.). The resulting solution was stirred overnight at room temperature and refluxed $\left(140^{\circ} \mathrm{C}\right)$ for 2 hours. After that time, the reaction was complete. The reaction solution was then cooled to room temperature, and ice water $(7 \mathrm{ml})$ was added into the solution at $-10^{\circ} \mathrm{C}$. A white precipitate formed. The solid was filtered, washed with cold water, and dried under reduced pressure. ${ }^{1} \mathrm{H}$ NMR analysis of the solid showed presence of ethylenediamine, which was responsible for the formation of an undesired byproduct in the next step. The solid was repurified chromatographically (CombiFlash, $\mathrm{SiO}_{2}, 100 \%$ EtOAc) to obtain (2-aminoethyl)chenodeoxycholic amide (CDCen) $(720 \mathrm{mg}, 62 \%) .{ }^{1} \mathrm{H}$ NMR (300 MHz, $d_{6}$-DMSO) $\delta 7.70$ $(\mathrm{t}, J=5.6,1 \mathrm{H}), 4.31(\mathrm{~d}, J=4.6,1 \mathrm{H}), 4.11(\mathrm{~d}, J=3.4,1 \mathrm{H}), 3.62(\mathrm{~s}, 1 \mathrm{H})$, $3.17(\mathrm{~s}, 1 \mathrm{H}), 3.00(\mathrm{q}, J=6.2,2 \mathrm{H}), 2.53(\mathrm{q}, J=6.7,2 \mathrm{H}), 2.34-1.54(\mathrm{~m}, 10 \mathrm{H})$, $1.53-0.85(\mathrm{~m}, 18 \mathrm{H}), 0.88(\mathrm{~d}, J=6.5,3 \mathrm{H}), 0.83(\mathrm{~s}, 3 \mathrm{H})$, and $0.60(\mathrm{~s}, 3 \mathrm{H})$. HRMS $m / z$ was calculated for $\mathrm{C}_{26} \mathrm{H}_{47} \mathrm{~N}_{2} \mathrm{O}_{3}[\mathrm{M}+\mathrm{H}]^{+} 435.3581$, which found $435.3582, \Delta 0.25 \mathrm{ppm}$. LC-MS $\mathrm{t}_{\mathrm{R}} 2.97$ minutes, $m / z$ $[\mathrm{M}+\mathrm{H}]^{+}$435.38.

CenNBD. 4-Chloro-7-nitrobenzofurazan ( $60 \mathrm{mg}, 0.3 \mathrm{mmol}, 1.5 \mathrm{eq}$.) was dissolved in anhydrous $N, N$-dimethylformamide (DMF) $(2 \mathrm{ml})$. After the addition of triethylamine $(60 \mu \mathrm{l}, 44 \mathrm{mg}, 0.44 \mathrm{mmol}, 2.2 \mathrm{eq}$.) and Cen (90 mg, $0.2 \mathrm{mmol}, 1$ eq.), the mixture was stirred at room temperature for 3 hours. The reaction solution was diluted with water $(30 \mathrm{ml})$ and extracted with ethyl acetate $(5 \times 15 \mathrm{ml})$. The organic layer was washed with water $(5 \times 10 \mathrm{ml})$ and then dried over $\mathrm{Na}_{2} \mathrm{SO}_{4}$. 
After evaporation under reduced pressure, a brown-green solid was obtained. The crude was purified by CombiFlash $\left(\mathrm{SiO}_{2}, 4 \mathrm{~g}, \mathrm{EtOAc} / \mathrm{MeOH}\right.$ 10:0 to 8:2, compounds eluted between $15 \%$ and $20 \% \mathrm{MeOH}$ ). The final compound was isolated as a yellow/reddish solid $(58 \mathrm{mg}, 48 \%) .{ }^{1} \mathrm{H}$ NMR $\left(300 \mathrm{MHz}, d_{6}\right.$-DMSO) $\delta 9.40(\mathrm{~s}, 1 \mathrm{H}), 8.53(\mathrm{~d}, J=8.9,1 \mathrm{H}), 8.00(\mathrm{t}$, $J=5.8,1 \mathrm{H}), 6.43(\mathrm{~d}, J=9.0,1 \mathrm{H}), 4.30(\mathrm{~d}, J=4.2,1 \mathrm{H}), 4.07(\mathrm{~d}, J=3.3$, $1 \mathrm{H}), 3.99(\mathrm{~d}, J=3.4,1 \mathrm{H}), 3.72(\mathrm{~d}, J=3.3,1 \mathrm{H}), 3.56(\mathrm{~m}, 3 \mathrm{H}), 3.39(\mathrm{q}, J=$ $5.7,1 \mathrm{H}), 3.17(\mathrm{~m}, 1 \mathrm{H}), 2.30-1.86(\mathrm{~m}, 6 \mathrm{H}), 1.86-1.50(\mathrm{~m}, 6 \mathrm{H}), 1.50-0.69$ $(\mathrm{m}, 16 \mathrm{H}), 0.87(\mathrm{~d}, J=6.1,3 \mathrm{H}), 0.79(\mathrm{~s}, 3 \mathrm{H})$, and $0.49(\mathrm{~s}, 3 \mathrm{H}) .{ }^{13} \mathrm{C} \mathrm{NMR}$ (75 MHz, $d_{6}$-DMSO) $\delta 173.40,145.36,144.39,144.06,137.85,120.82$, 99.11, 70.94, 70.40, 66.20, 48.56, 46.04, 45.65, 43.26, 41.49, 41.27, $39.44,35.27,34.95,34.86,34.34,32.42,31.46,30.37,28.50,27.18$, $26.31,26.17,22.70,22.55,17.06$, and 12.22 . HRMS $m / z$ was calculated for $\mathrm{C}_{32} \mathrm{H}_{48} \mathrm{~N}_{5} \mathrm{O}_{7}[\mathrm{M}+\mathrm{H}]^{+} 614.3548$, which found $614.3554, \Delta 0.97 \mathrm{ppm}$. LC-MS $t_{R} 3.57$ minutes, $99 \%$ pure (at $\lambda_{214 n m}$ ). UV-visible/Fluo (PBS buffer $+1 \%$ DMSO): $\lambda_{\text {max abs }} 478 \mathrm{~nm}, \lambda_{\max \text { em }} 545 \mathrm{~nm}$.

CDCenNBD. Analogously to CenNBD, the title compound was prepared from 4-chloro-7-nitrobenzofurazan ( $60 \mathrm{mg}, 0.3 \mathrm{mmol}, 1.5$ eq.) and CDCen ( $86 \mathrm{mg}, 0.2 \mathrm{mmol}, 1$ eq.) in the presence of triethylamine (60 $\mu \mathrm{l}, 44 \mathrm{mg}, 0.44 \mathrm{mmol}, 2.2 \mathrm{eq}$.) in DMF ( $2 \mathrm{ml})$. The crude was purified twice by CombiFlash $\left(\mathrm{SiO}_{2}, 4 \mathrm{~g}\right.$, EtOAc/MeOH 10:0 to 8:2) to obtain the final compound as a yellow/reddish solid (50 mg, 42\%). ${ }^{1} \mathrm{H}$ NMR $\left(300 \mathrm{MHz}, d_{6}\right.$-DMSO) $\delta 9.40(\mathrm{~s}, 1 \mathrm{H}), 8.53(\mathrm{~d}, J=8.9,1 \mathrm{H})$, $8.01(\mathrm{t}, J=5.7,1 \mathrm{H}), 6.43(\mathrm{~d}, J=9.0,1 \mathrm{H}), 4.30(\mathrm{~d}, J=4.6,1 \mathrm{H}), 4.09$ $(\mathrm{d}, J=3.4,1 \mathrm{H}), 3.56(\mathrm{~m}, 3 \mathrm{H}), 3.38(\mathrm{q}, J=5.8,2 \mathrm{H}), 3.25-3.10(\mathrm{~m}, 1 \mathrm{H})$, $2.33-1.52(\mathrm{~m}, 12 \mathrm{H}), 1.52-0.72(\mathrm{~m}, 24 \mathrm{H})$, and $0.51(\mathrm{~s}, 3 \mathrm{H}) .{ }^{13} \mathrm{C} \mathrm{NMR}$ $\left(101 \mathrm{MHz}, d_{4}\right.$-MeOD) $\delta 177.71,146.83,145.80,145.39,138.47,123.30$, $99.95,72.84,69.03,57.20,51.42,44.68,43.59,43.11,40.90,40.70$, $40.41,39.08,36.71,36.51,36.16,35.83,34.07,33.97,33.12,31.32$, 29.17, 24.53, 23.36, 21.70, 18.89, and 12.12. HRMS $m / z$ was calculated for $\mathrm{C}_{32} \mathrm{H}_{48} \mathrm{~N}_{5} \mathrm{O}_{6}[\mathrm{M}+\mathrm{H}]^{+} 598.3599$, which found 598.3592, $\Delta-1.24 \mathrm{ppm}$. LC-MS $t_{R} 3.86$ minutes, $95 \%$ pure (at $\lambda_{214 \mathrm{~nm}}$ ).

AcenNBD. The same procedure was used as for CenNBD above. 4-Chloro-7-nitrobenzofurazan (293 mg, $1.47 \mathrm{mmol}, 1.47 \mathrm{eq}$.) and $N$-(2aminoethyl) acetamide (95 $\mu \mathrm{l}, 102 \mathrm{mg}, 1 \mathrm{mmol}, 1$ eq.) reacted in the presence of triethylamine ( $300 \mu \mathrm{l}, 218 \mathrm{mg}, 2.15 \mathrm{mmol}, 2.2 \mathrm{eq}$.) in DMF $(10 \mathrm{ml})$. The crude was purified by CombiFlash $\left(\mathrm{SiO}_{2}, 12 \mathrm{~g}\right.$, EtOAc/MeOH 10:0 to 8:2). Only the purest fractions were collected, as many were contaminated with traces of a fluorescent byproduct, and the final compound was isolated as a yellow/reddish solid (22 mg, 8\%). ${ }^{1} \mathrm{H}$ NMR (300 MHz, $d_{6}$-DMSO) $\delta 9.44(\mathrm{br} \mathrm{s}, 1 \mathrm{H}), 8.54(\mathrm{~d}, J=8.9,1 \mathrm{H}), 8.07(\mathrm{t}, J=$ $5.9,1 \mathrm{H}), 6.43(\mathrm{~d}, J=9.0,1 \mathrm{H}), 3.53(\mathrm{br} \mathrm{s}, 2 \mathrm{H}), 3.35(\mathrm{q}, J=6.1,2 \mathrm{H})$, and $1.80(\mathrm{~s}, 3 \mathrm{H}) .{ }^{13} \mathrm{C}$ NMR (101 MHz, $d_{6}$-DMSO) $\delta 169.94,145.37,144.46$, 144.10, 137.93, 120.86, 99.12, 43.10, 37.28, and 22.58. HRMS $m / z$ was calculated for $\mathrm{C}_{10} \mathrm{H}_{11} \mathrm{~N}_{5} \mathrm{O}_{4}[\mathrm{M}+\mathrm{H}]^{+} 266.0884$, which found 266.0884, $\Delta 0.04 \mathrm{ppm}$. LC-MS $\mathrm{t}_{\mathrm{R}} 2.02$ minutes, $>90 \%$ pure (at $\lambda_{214 \mathrm{~nm}}$ ).

CenDNS. Dansyl chloride (30 mg, $0.11 \mathrm{mmol}, 1.1$ eq.) was dissolved in dichloromethane (DCM) $(1 \mathrm{ml})$ and added to Cen (45 $\mathrm{mg}, 0.1 \mathrm{mmol}, 1$ eq. $)$ in DCM $(1 \mathrm{ml})$ at $0^{\circ} \mathrm{C}$, which was followed by the addition of triethylamine ( $30 \mu \mathrm{l}, 22 \mathrm{mg}, 0.22 \mathrm{mmol}, 2.2 \mathrm{eq}$.). The resulting solution was stirred overnight while slowly warming up to room temperature. After 18 hours, the reaction solution was diluted with aq. $\mathrm{NaHCO}_{3}(5 \mathrm{ml})$ and extracted with ethyl acetate $(3 \times 25 \mathrm{ml})$, and the organic layer was dried over $\mathrm{Na}_{2} \mathrm{SO}_{4}$ and evaporated under reduced pressure. The crude was purified by CombiFlash $\left(\mathrm{SiO}_{2}, 12 \mathrm{~g}\right.$, EtOAc/MeOH 10:0 to 8:2). The title compound was obtained as pale yellow solid (51 mg, 75\%). ${ }^{1} \mathrm{H}$ NMR $\left(300 \mathrm{MHz}, d_{6}\right.$-DMSO) $\delta 8.46(\mathrm{dt}, J=$ $8.5,1.1,1 \mathrm{H}), 8.26(\mathrm{~d}, J=8.6,1 \mathrm{H}), 8.08(\mathrm{dd}, J=7.3,1.2,1 \mathrm{H}), 7.94(\mathrm{t}, J=$ $5.8,1 \mathrm{H}), 7.69(\mathrm{t}, J=5.7,1 \mathrm{H}), 7.60(\mathrm{~m}, 2 \mathrm{H}), 7.26(\mathrm{dd}, J=7.7,0.9,1 \mathrm{H})$, $4.31(\mathrm{~d}, J=4.3,1 \mathrm{H}), 4.07(\mathrm{~d}, J=3.6,1 \mathrm{H}), 3.99(\mathrm{~d}, J=3.4,1 \mathrm{H}), 3.75(\mathrm{~d}$, $J=3.4,1 \mathrm{H}), 3.60(\mathrm{br} \mathrm{s}, 1 \mathrm{H}), 3.25-3.10(\mathrm{~m}, 1 \mathrm{H}), 3.02(\mathrm{q}, J=6.3,2 \mathrm{H}), 2.83$ (s, 6H), $2.76(\mathrm{q}, J=6.2,2 \mathrm{H}), 2.37-0.76(\mathrm{~m}, 24 \mathrm{H}), 0.87(\mathrm{~d}, J=6.2,3 \mathrm{H})$, $0.81(\mathrm{~s}, 3 \mathrm{H})$, and $0.53(\mathrm{~s}, 3 \mathrm{H}) .{ }^{13} \mathrm{C} \mathrm{NMR}\left(101 \mathrm{MHz}, d_{4}-\mathrm{MeOD}\right) \delta 177.16$, 153.29, 136.74, 131.29 (2C), 130.92, 130.29, 129.21, 124.31, 120.45, 116.47, 74.04, 72.89, 69.05, 48.00, 47.48, $45.81\left(2 \mathrm{C} \mathrm{N}\left(\mathrm{CH}_{3}\right)_{2}\right), 43.34$, $43.22,43.00,41.03,40.49,40.28,36.83,36.50,35.91,33.98,33.07$, $31.20,30.73,29.60,28.66,27.89,24.21,23.17,17.74$, and $12.99 .{ }^{13} \mathrm{C}$ NMR (101 MHz, $d_{6}$-DMSO) $\delta 172.76,151.35,135.73,129.42,129.05$,
$128.98,128.26,127.83,123.52,118.97,115.11,70.95,70.39,66.20$, $48.55,46.03,45.67,45.03,41.95,41.49,41.32$, 38.46, 35.28, 35.01, $34.86,34.35,32.37,31.47,30.37,28.52,27.22$, 26.18, 22.73, 22.59, 17.05 , and 12.26 (one signal overlapped with the solvent signal). HRMS $m / z$ was calculated for $\mathrm{C}_{38} \mathrm{H}_{58} \mathrm{~N}_{3} \mathrm{O}_{6} \mathrm{~S}[\mathrm{M}+\mathrm{H}]^{+} 684.4041$, which found 684.4043, $\Delta 0.32 \mathrm{ppm}$. LC-MS $\mathrm{t}_{\mathrm{R}} 3.48$ minutes, $97 \%$ pure (at $\left.\lambda_{214 \mathrm{~nm}}\right)$. UV-visible/Fluo $(\mathrm{MeOH}+1 \% \mathrm{DMSO}): \lambda_{\max }$ abs $334 \mathrm{~nm}$, $\lambda_{\text {max em }} 520 \mathrm{~nm}$.

CDCenDNS. The procedure above was used for the synthesis of CDCenDNS, starting from dansyl chloride ( $60 \mathrm{mg}, 0.22 \mathrm{mmol}, 1.1$ eq.) and CDCen ( $86 \mathrm{mg}, 0.2 \mathrm{mmol}, 1$ eq.) in DCM ( $2 \mathrm{ml})$ and triethylamine $(60 \mu \mathrm{l}, 44 \mathrm{mg}, 0.44 \mathrm{mmol}, 2.2 \mathrm{eq}$.). After purification by CombiFlash $\left(\mathrm{SiO}_{2}, 4 \mathrm{~g}, \mathrm{EtOAc} / \mathrm{MeOH}\right.$ 10:0 to 8:2), the final compound was isolated as a pale yellow solid ( $81 \mathrm{mg}, 61 \%) .{ }^{1} \mathrm{H}$ NMR (300 MHz, $d_{6}$-DMSO) $\delta$ $8.46(\mathrm{~d}, J=8.5,1 \mathrm{H}), 8.26(\mathrm{~d}, J=8.7,1 \mathrm{H}), 8.08(\mathrm{dd}, J=7.3,1.3,1 \mathrm{H}), 7.94$ $(\mathrm{t}, J=5.9,1 \mathrm{H}), 7.69(\mathrm{t}, J=5.7,1 \mathrm{H}), 7.60(\mathrm{~m}, 2 \mathrm{H}), 7.26(\mathrm{~d}, J=7.4,1 \mathrm{H})$, $4.31(\mathrm{~d}, J=4.7,1 \mathrm{H}), 4.10(\mathrm{~d}, J=3.5,1 \mathrm{H}), 3.62(\mathrm{~s}, 1 \mathrm{H}), 3.02(\mathrm{q}, J=6.5$, $2 \mathrm{H}), 2.83(\mathrm{~s}, 6 \mathrm{H}), 2.76$ (q, $J=6.6,2 \mathrm{H}), 2.19$ (q, $J=12.9,1 \mathrm{H}), 2.07-0.66$ $(\mathrm{m}, 35 \mathrm{H})$, and $0.54(\mathrm{~s}, 3 \mathrm{H}) .{ }^{13} \mathrm{C} \mathrm{NMR}\left(75 \mathrm{MHz}, d_{4}-\mathrm{MeOD}\right) \delta 177.07$, $153.17,136.67,131.25,131.17,130.86,130.25,129.19,124.29,120.40$, 116.37, 72.80, 68.96, 57.15, 51.46, 45.82, 43.60, 43.32, 43.10, 40.98, $40.70,40.41,40.37,36.77,36.53,36.17,35.87,33.98,33.94,33.04$, $31.34,29.21,24.58,23.43,21.75,18.94$, and 12.22 . HRMS $m / z$ was calculated for $\mathrm{C}_{38} \mathrm{H}_{58} \mathrm{~N}_{3} \mathrm{O}_{5} \mathrm{~S}[\mathrm{M}+\mathrm{H}]^{+}$668.4092, which found 668.4080, $\Delta-1.72 \mathrm{ppm}$. LC-MS $\mathrm{t}_{\mathrm{R}} 3.80$ minutes, $99 \%$ pure (at $\lambda_{214 \mathrm{~nm}}$ ).

AcenDNS. This control compound was prepared similarly to CenDNS and CDCenDNS from $N$-(2-aminoethyl) acetamide (95 $\mu \mathrm{l}, 102 \mathrm{mg}$, $1 \mathrm{mmol}, 1$ eq.) and dansyl chloride (290 mg, $1.077 \mathrm{mmol}, 1.077$ eq.) in $\operatorname{DCM}(7.5 \mathrm{ml})$ and triethylamine (300 $\mu \mathrm{l}, 218 \mathrm{mg}, 2.15 \mathrm{mmol}, 2.2$ eq.). The crude was purified by CombiFlash $\left(\mathrm{SiO}_{2}, 12 \mathrm{~g}\right.$, EtOAc/MeOH 10:0 to 8:2) to obtain AcenDNS as a pale yellow solid (275 mg, 82\%). ${ }^{1} \mathrm{H}$ NMR (300 MHz, $d_{6}$-DMSO) $\delta 8.47(\mathrm{~d}, J=8.5,1 \mathrm{H}), 8.28(\mathrm{~d}, J=8.7,1 \mathrm{H})$, $8.10(\mathrm{dd}, J=7.3,1.2,1 \mathrm{H}), 7.96(\mathrm{br} \mathrm{s}, 1 \mathrm{H}), 7.77(\mathrm{t}, J=5.6,1 \mathrm{H}), 7.63$ (dd, $J=8.6,7.3,1 \mathrm{H}), 7.60(\mathrm{dd}, J=8.5,7.7,1 \mathrm{H}), 7.27(\mathrm{dd}, J=7.6,0.9,1 \mathrm{H})$, 3.09-2.93 [m (pseudo q), $2 \mathrm{H}$ ], $2.84(\mathrm{~s}, 6 \mathrm{H}), 2.79(\mathrm{t}, J=6.9,2 \mathrm{H})$, and 1.69 (s, 3H). ${ }^{13} \mathrm{C}$ NMR (101 MHz, $d_{6}$-DMSO) $\delta 169.32,151.37,135.75$, $129.45,129.07,129.00,128.30,127.86,123.58,119.03,115.14,45.06$, $41.91,38.62$, and 22.45. HRMS $m / z$ was calculated for $\mathrm{C}_{16} \mathrm{H}_{21} \mathrm{~N}_{3} \mathrm{O}_{3} \mathrm{~S}$ $[\mathrm{M}+\mathrm{H}]^{+} 336.1376$, which found $336.1370, \Delta-1.90$ ppm. LC-MS $t_{R}$ 2.32 minutes, $>90 \%$ pure (at $\lambda_{214 n m}$ ).

CenPB. Amine Cen (13 mg, $0.029 \mathrm{mmol}, 1$ eq.) was dissolved in DMF (0.8 ml). Diisopropylethylamine $(100 \mu \mathrm{l}, 76 \mathrm{mg}, 0.59 \mathrm{mmol}$, 20 eq.) was added, which was followed by Pacific Blue succinimidyl ester (10 mg, $0.029 \mathrm{mmol}, 1$ eq.). The mixture was stirred for 3 hours at room temperature, after which the reaction mixture was evaporated under reduced pressure. The crude was purified by column chromatography $\left(\mathrm{SiO}_{2}\right.$, EtOAc/MeOH 8:2), and the obtained compound was recrystallized from water/MeOH (4:1). The pale yellow solid was filtered, washed with cold water, and dried in vacuo to yield CenPB (11 mg, 55\%). ${ }^{1} \mathrm{H}$ NMR (300 MHz, $d_{6}$-DMSO) $\delta 11.95(\mathrm{br} \mathrm{s}, 1 \mathrm{H})$ $8.63(\mathrm{~m}, 2 \mathrm{H}), 7.86(\mathrm{t}, J=5.3,1 \mathrm{H}), 7.51(\mathrm{~d}, J=10.9,1 \mathrm{H}), 4.29(\mathrm{br} \mathrm{s}, 1 \mathrm{H})$, $4.06(\mathrm{~m}, 2 \mathrm{H}), 3.97(\mathrm{br} \mathrm{s}, 1 \mathrm{H}), 3.72(\mathrm{~d}, J=3.4,1 \mathrm{H}), 3.56(\mathrm{br} \mathrm{s}, 1 \mathrm{H})$, 3.4-3.11 ( $\mathrm{m}, 4 \mathrm{H}$, partially overlapped with the solvent signal), $2.34-1.85(\mathrm{~m}, 6 \mathrm{H}), 1.86-1.53(\mathrm{~m}, 6 \mathrm{H}), 1.48-1.02(\mathrm{~m}, 10 \mathrm{H}), 1.02-0.73$ $(\mathrm{m}, 2 \mathrm{H}), 0.89(\mathrm{~d}, J=6.2,3 \mathrm{H}), 0.77(\mathrm{~s}, 3 \mathrm{H})$, and $0.48(\mathrm{~s}, 3 \mathrm{H}) .{ }^{13} \mathrm{C} \mathrm{NMR}$ $\left(101 \mathrm{MHz}, d_{6}\right.$-DMSO) $\delta 173.34,162.68,160.82,147.69,110.30(\mathrm{~d}, J=$ 22.5), 71.48, 70.93, 66.71, 49.07, 46.71, 46.18, 42.02, 41.75, 39.28, $38.77,35.78,35.43,35.34,34.84,33.06,32.09$, 30.88, 28.96, 27.72, $26.65,23.23,23.01,17.57,12.72 .{ }^{19} \mathrm{~F} \mathrm{NMR}\left(282 \mathrm{MHz}, d_{6}\right.$-DMSO) $\delta$ -135.66 (br s). HRMS $m / z$ was calculated for $\mathrm{C}_{36} \mathrm{H}_{49} \mathrm{~F}_{2} \mathrm{~N}_{2} \mathrm{O}_{8}[\mathrm{M}+\mathrm{H}]^{+}$ 675.3451 , which found $675.3453, \Delta 0.24 \mathrm{ppm}$. LC-MS $\mathrm{t}_{\mathrm{R}} 3.23$ minutes, $97 \%$ pure (at $\lambda_{214 \mathrm{~nm}}$ ). UV-visible/Fluo (PBS buffer $+1 \%$ DMSO): $\lambda_{\max \text { abs }} 410 \mathrm{~nm}, \lambda_{\max \text { em }} 451 \mathrm{~nm}$.

CDCenPB. Analogously to above, CDCen (50 mg, $0.115 \mathrm{mmol}, 1$ eq.) was dissolved in DMF (2 ml). Diisopropylethylamine (200 $\mu \mathrm{l}, 152$ $\mathrm{mg}, 1.176 \mathrm{mmol}, 10 \mathrm{eq}$.) was added, which was followed by Pacific Blue succinimidyl ester ( $39 \mathrm{mg}, 0.115 \mathrm{mmol}, 1$ eq.). The mixture was stirred for 4 hours at room temperature, and the reaction mixture was 
evaporated under reduced pressure. The crude was purified by CombiFlash $\left(\mathrm{SiO}_{2}, 4 \mathrm{~g}, \mathrm{EtOAc} / \mathrm{MeOH}\right.$ 10:0 to 8:2) to give CDCenPB (38 mg, 50\%). ${ }^{1} \mathrm{H}$ NMR (300 MHz, $d_{6}$-DMSO) $\delta 8.69(\mathrm{~d}, J=1.6,1 \mathrm{H})$, $8.64(\mathrm{t}, J=5.7,1 \mathrm{H}), 7.87(\mathrm{t}, J=5.4,1 \mathrm{H}), 7.59(\mathrm{~d}, J=10.7,1 \mathrm{H}), 4.29(\mathrm{br} \mathrm{s}$, $1 \mathrm{H}), 4.13-4.01(\mathrm{~m}, 2 \mathrm{H}), 3.57$ (br s, $1 \mathrm{H}), 3.44-3.10$ (m, 4H, partially overlapped with the solvent signal), $2.33-0.85(\mathrm{~m}, 26 \mathrm{H}), 0.85(\mathrm{~d}, J=$ $6.3,3 \mathrm{H}), 0.80(\mathrm{~s}, 3 \mathrm{H})$, and $0.49(\mathrm{~s}, 3 \mathrm{H}) .{ }^{13} \mathrm{C} \mathrm{NMR}\left(101 \mathrm{MHz}, d_{4}-\mathrm{MeOD}\right) \delta$ $177.17,164.63,162.00,152.24(\mathrm{~d}, J=243.0$ ), 149.23, 147.00, 142.68 (dd, $J=38.0,7.9), 140.06(\mathrm{~d}, J=6.5), 114.30,110.89(\mathrm{~d}, J=21.3)$, 109.17-108.68 (m), 72.86, 69.01, 57.40, 51.43, 43.61, 43.16, 40.96, $40.74,40.45,40.29,39.88,36.72,36.53,36.17,35.87,34.21,33.96$, $33.21,31.33,29.21,24.57,23.33,21.71,18.91$, and 12.17. ${ }^{19} \mathrm{~F}$ NMR (282 MHz,$d_{6}$-DMSO) $\delta-135.48$ (br s). HRMS $m / z$ was calculated for $\mathrm{C}_{36} \mathrm{H}_{48} \mathrm{~F}_{2} \mathrm{~N}_{2} \mathrm{NaO}_{7}[\mathrm{M}+\mathrm{Na}]^{+} 681.3322$, which found 681.3309, $\Delta-1.86$ ppm. LC-MS $t_{\mathrm{R}} 3.53$ minutes, $98 \%$ pure (at $\lambda_{214 \mathrm{~nm}}$ ).

AcenPB. As above, $N$-(2-aminoethyl) acetamide $(20 \mu \mathrm{l}, 21 \mathrm{mg}$, $0.209 \mathrm{mmol}, 1.77$ eq.) and Pacific Blue succinimidyl ester $(40 \mathrm{mg}$, $0.118 \mathrm{mmol}, 1$ eq.) reacted in the presence of diisopropylethylamine (100 $\mu$ l, $76 \mathrm{mg}, 0.59 \mathrm{mmol}, 20$ eq.) in DMF. The crude was purified by CombiFlash $\left(\mathrm{SiO}_{2}, 4 \mathrm{~g}\right.$, EtOAc/MeOH 10:0 to 8:2) to obtain AcenPB as a pale yellow solid $(25 \mathrm{mg}, 68 \%) .{ }^{1} \mathrm{H}$ NMR $(300 \mathrm{MHz}$, $d_{6}$-DMSO) $\delta 12.10(\mathrm{~s}, 1 \mathrm{H}), 8.70(\mathrm{~d}, J=1.6,1 \mathrm{H}), 8.66(\mathrm{t}, J=5.8,1 \mathrm{H})$, $7.96(\mathrm{t}, J=5.5,1 \mathrm{H}), 7.61(\mathrm{~d}, J=11.1), 3.36(\mathrm{q}, J=6.1,2 \mathrm{H}), 3.19$ (q, $J=6.1,2 \mathrm{H})$, and $1.80(\mathrm{~s}, 3 \mathrm{H}) .{ }^{13} \mathrm{C} \mathrm{NMR}\left(101 \mathrm{MHz}, d_{6}\right.$-DMSO) $\delta$ $169.49,161.97,160.12,150.40(\mathrm{dd}, J=240.9,5.5), 147.27$ (t, $J=3.3$ ), 146.29-145.41 (m), $141.28(\mathrm{~d}, J=8.4), 139.56(\mathrm{dd}, J=242.1,7.9)$, 112.06, 110.06 (dd, $J=21.1,3.0), 106.66-105.90(\mathrm{~m}), 38.86,38.36$, and $22.60 .{ }^{19} \mathrm{~F}$ NMR $\left(282 \mathrm{MHz}, d_{6}\right.$-DMSO) $\delta-135.47(\mathrm{t}, J=11.3)$. HRMS $m / z$ was calculated for $\mathrm{C}_{14} \mathrm{H}_{12} \mathrm{~F}_{2} \mathrm{~N}_{2} \mathrm{O}_{5}[\mathrm{M}+\mathrm{H}]^{+} 327.0787$, which found 327.0789, $\Delta 0.72 \mathrm{ppm}$. LC-MS $\mathrm{t}_{\mathrm{R}} 1.94$ minutes, $>90 \%$ pure (at $\lambda_{214 \mathrm{~nm}}$ ).

\section{Results}

Synthesis of Fluorescent Bile Salt Probes. Previous examples of fluorescent BS derivatives indicate that the carboxylic acid side chain at C-24 of the bile acid skeleton is tolerant toward substitution with fluorescent dyes and other moieties (Holzinger et al., 1997; Yamaguchi et al., 2006; Rais et al., 2010; Dong et al., 2015; Testa et al., 2017). In addition, we have decided to introduce a short ethylene diamine linker unit to mimic the amino acids glycine and taurine, which are commonly conjugated at this position in human bile acids (Fig. 1A). With regards to the choice of fluorescent dyes, we reasoned that small organic fluorescent dyes would interfere the least with the transport mechanisms, and therefore we conjugated NBD, DNS, and PB to the C-24 position of cholic and chenodeoxycholic acid (Fig. 1B). Furthermore, these three dyes could potentially mimic certain features of the natural transport substrates; NBD and DNS both have slightly acidic $\mathrm{NH}$ groups, the sulfonamide group of DNS is similar to the sulfonic acid end group of taurine, and, due to the two strongly electron-withdrawing fluorine atoms, the phenol group of PB is always deprotonated under physiologic conditions like the end groups of human bile acids (Fig. 1A).

The synthesis of the fluorescent BS derivatives started from cholic or chenodeoxycholic acid, which were transformed to the corresponding methyl esters (Scheme 1A). Aminolysis in refluxing ethylenediamine yielded the two amide building blocks Cen and CDCen. It is important at this point to quantitatively remove any remaining ethylenediamine. The three fluorescent dyes were then coupled to the primary amine terminus of Cen and CDCen using standard conditions and commercial dye reagents NBD chloride, dansyl chloride, and Pacific Blue $N$-hydroxysuccinimid ester. The isolated final probes were subjected to a number of purification steps, including washing, precipitation, and chromatographic methods, to ensure that all traces of uncoupled fluorescent dyes were removed. This is crucial, as several studies in the past have shown that fluorescent dyes alone can also act as transport substrates (De Bruyn et al., 2011; Patik et al., 2015, 2018). Consequently, our extensive purification efforts delivered the final fluorescent BS probes in high purity ( $>95 \%$ according to NMR and high-pressure liquid chromatography) albeit at the expense of isolated yields $(42-75 \%)$. In a similar fashion, we have also generated truncated control compounds AcenNBD, AcenDNS, and AcenPB, which lack the bile acid moiety. All final compounds showed strong fluorescence emission in the region of $450-550 \mathrm{~nm}$ (Scheme 1B).

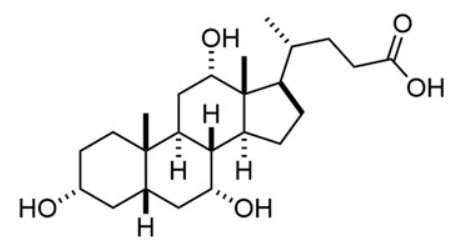

Cholic acid (CA)<smiles>C[C@H](CCC(=O)NCCS(=O)(=O)O)C1CC[C@H]2[C@H]3[C@H](C[C@H](O)[C@]12C)C1(C)CC[C@H](O)C[C@H]1C[C@H]3O</smiles>

Taurocholic acid (TCA)

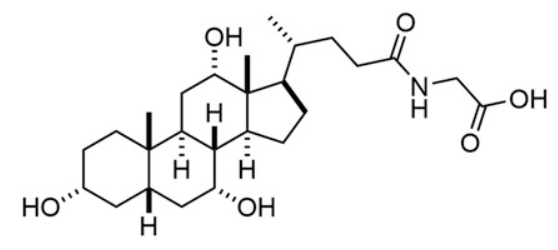

Glycocholic acid (GCA)<smiles>C[C@H](CCC(=O)NCC(=O)O)C1CC[C@H]2[C@H]3C(CCC12C)C1(C)CC[C@H](O)C[C@H]1C[C@H]3O</smiles>

Fig. 1. (A) Structures of common human bile salts. (B) Structures of synthetic fluorescent bile salt derivatives. 


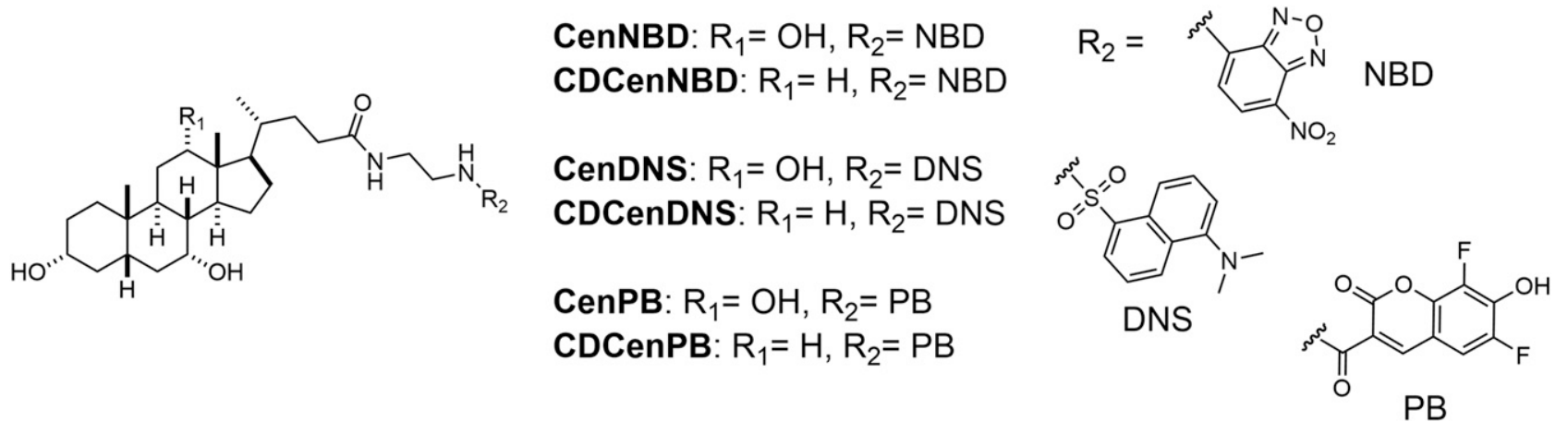

Fig. 1. Continued.

Generation of CHO-ASBT Cells. To demonstrate the successful transfection of CHO cells with functional ASBT, we determined their capacity for sodium-dependent BS uptake. Figure 2A shows sodium-dependent uptake into transfected $\mathrm{CHO}$ cells, which is absent in wild-type $\mathrm{CHO}$ cells. Figure 2B confirms expression of ASBT by Western blotting and expression at the plasma membrane of transfected CHO cells by immunofluorescence, which is again absent in wild-type cells. Hence, we successfully established an ASBT-expressing CHO cell line. For a final characterization of this novel cell line, we determined the concentration dependence of sodium-dependent uptake of taurocholate under initial uptake conditions. Figure 3 shows that the $K_{\mathrm{m}}$ value is about $34 \mu \mathrm{M}$, which is in line with published values (Craddock et al., 1998).

Inhibition of Transporters by Fluorescent Bile Salt Probes. To test the different fluorescent BS derivatives as potential substrates of BS and organic anion transporters, we chose a two-tier approach. First, the new BS derivatives were tested as potential inhibitors of a radioactively labeled standard substrate for the respective carrier. Derivatives

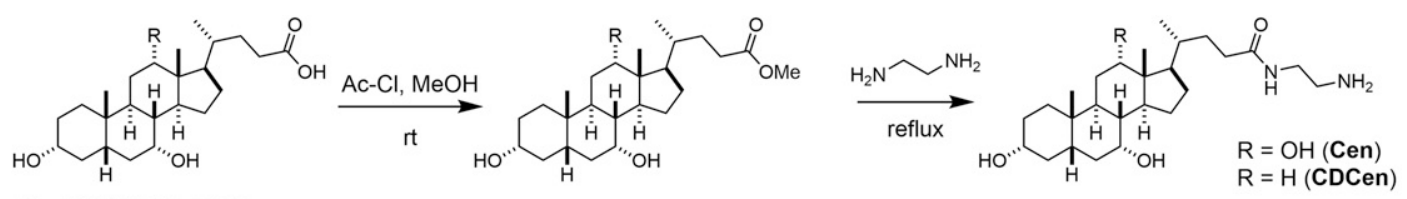

$\mathrm{R}=\mathrm{OH}$ (Cholic acid)

$\mathrm{R}=\mathrm{H}$ (Chenodeoxycholic acid)

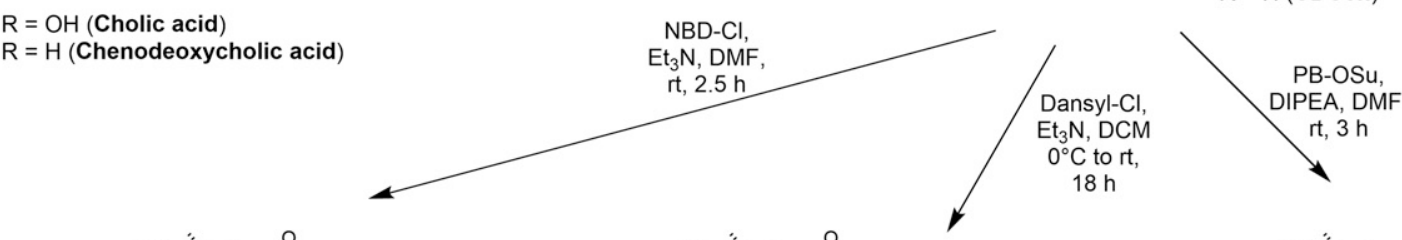

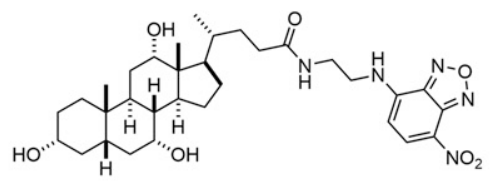

CenNBD

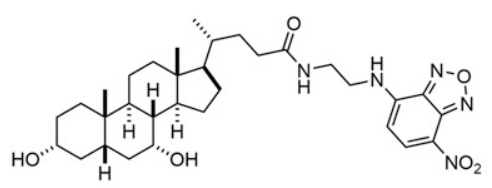

CDCenNBD<smiles>CC(=O)NCCNc1ccc(O)c2nonc12</smiles>

AcenNBD

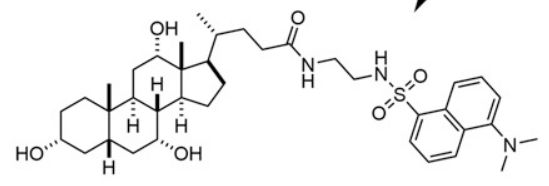

CenDNS

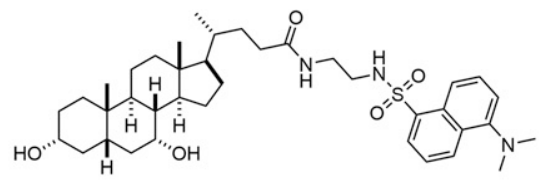

CDCenDNS

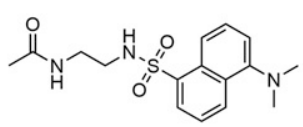

AcenDNS

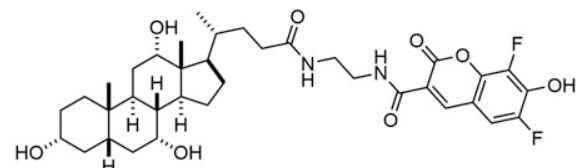

CenPB

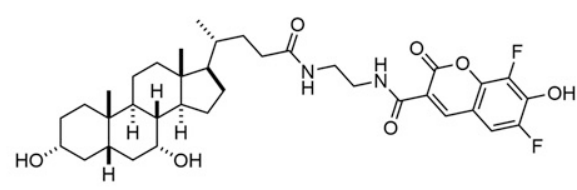

CDCenPB

Scheme 1. (A) Chemical synthesis of fluorescent bile salt derivatives. Reagents: Ac-Cl, acetyl chloride; Et ${ }_{3} \mathrm{~N}$, triethylamine; DMF; DCM; PB-OSu, PB $N$-hydroxysuccinimid ester; DIPEA, $N, N$-diisopropylethylamine. (B) Absorption and emission properties of fluorescent bile salt derivatives. Normalized UV-visible absorption and fluorescence emission spectra, CenNBD: $\lambda_{\max }$ abs $478 \mathrm{~nm}, \lambda_{\max }$ em $545 \mathrm{~nm}$; CenDNS: $\lambda_{\max }$ abs $334 \mathrm{~nm}, \lambda_{\max }$ em $520 \mathrm{~nm}$; CenPB: $\lambda_{\max }$ abs $410 \mathrm{~nm}, \lambda_{\max }$ em $451 \mathrm{~nm}$. TLC, spotted compounds run on thin-layer chromatography plates (silica gel; ethyl acetate/methanol 8:2) and excited at $366 \mathrm{~nm}$. Note the difference in polarity between CenDNS and CenPB. 
UV-Vis/fluo

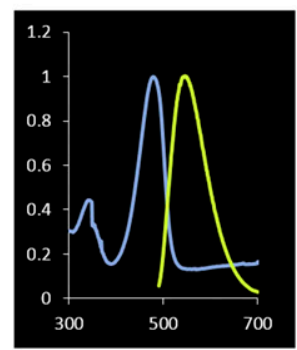

CenNBD
TLC

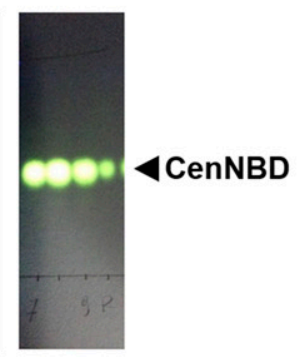

1

.

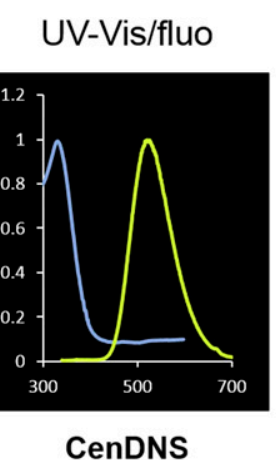

CenDNS

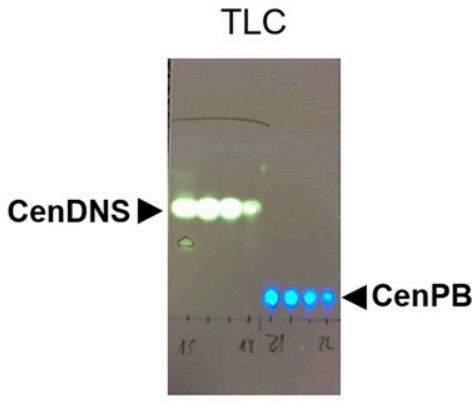

Scheme 1B
UV-Vis/fluo

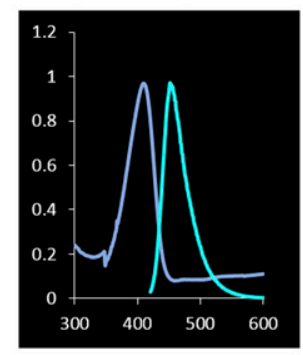

CenPB

Scheme 1. Continued.

inhibiting transport were secondly tested as potential substrates by performing a transport experiment. Table 1 lists the uptake of standard substrates for all transporters investigated. Comparing transport under control and transportermediated conditions shows for all transporters a transporterto-control ratio of more than 2.5 , which makes the test systems amenable for the inhibition studies. Figure 4 shows a typical inhibition experiment with an uptake transporter. This figure clearly demonstrates the concentration-dependent inhibition of OATP1B1-mediated estrone-3-sulfate by CenNBD. It should also be noted, that CenNBD did not affect the plasma membrane barrier function of the cells, as even at $100 \mu \mathrm{M}$ no increase of estrone-3-sulfate uptake into WT cells was found. Figure 5 gives an example of the efflux transporter BSEP. Here, ATP-dependent uptake of taurocholate was inhibited by CDCenNBD in a concentration-dependent manner. Similarly, no impact on vesicle membrane barrier function was found, as evidenced by an unchanged taurocholate transport in the absence of ATP. Because of the number of compounds to be tested on a large number of transporters, the experiments were not designed to obtain $\mathrm{IC}_{50}$ values for inhibiting fluorescent BS derivatives. Table 2 shows the summary of the inhibition experiments with uptake transporters. The pattern of inhibition is somewhat unexpected, as NTCP is strongly inhibited in a concentration-dependent manner by all fluorescent BS derivatives with the exception of CDCenDNS, which inhibits up to $\sim 55 \%$ at $100 \mu \mathrm{M}$. This

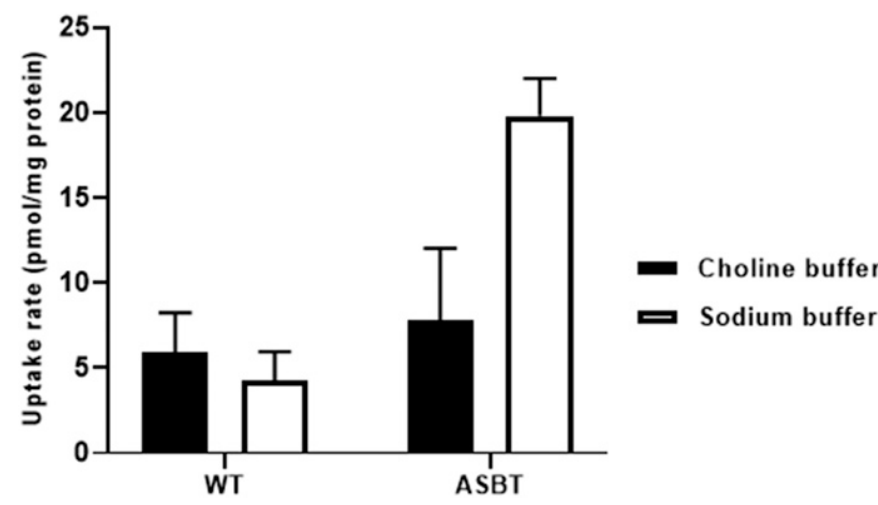

Fig. 2. Characterization of ASBT-expressing CHO cells. (A) Uptake of 5 $\mu \mathrm{M}$ taurocholate in WT and ASBT-expressing cells was measured for 3 minutes in the presence or absence of sodium as described in Materials and Methods. (B) Left: Western blot of WT and ASBT-expressing cells. The band for ASBT is indicated by an arrow. Right: immunolocalization of ASBT at the plasma membrane of transfected cells (upper panel). finding contrasts to some extent with the known restricted substrate specificity of NCTP (Stieger, 2011). Among the OATPs, the pattern is different and transporter-specific: Strong inhibition was observed for CenNBD against OATP1B3 and OATP2B1; CenDNS against OATP1B1 and OATP2B1; CenPB against OATP1B3; and CDCenPB against OATP1B1, OATP1B3, and OATP2B1. A weaker inhibition was found for CenNBD against OATP1B1, CenDNS against OATP1B3, CenPB against OATP1B1 and OATP2B1, CDCenNBD against OATP1B3 and OATP2B1, and CDCenDNS against OATP1B3 and OATP2B1. No relevant inhibition was observed for CDCenNBD against OATP1B1 and CDCenDNS against OATP1B1. Given the known broad substrate specificity of OATPs (Obaidat et al., 2012; Hagenbuch and Stieger, 2013), a good inhibition of most OATPs was anticipated. Finally, we also tested the intestinal BS transporter ASBT. Unexpectedly, not one of the derivatives was a strong inhibitor: With the exception of CDCenDNS and CDCenNBD (no

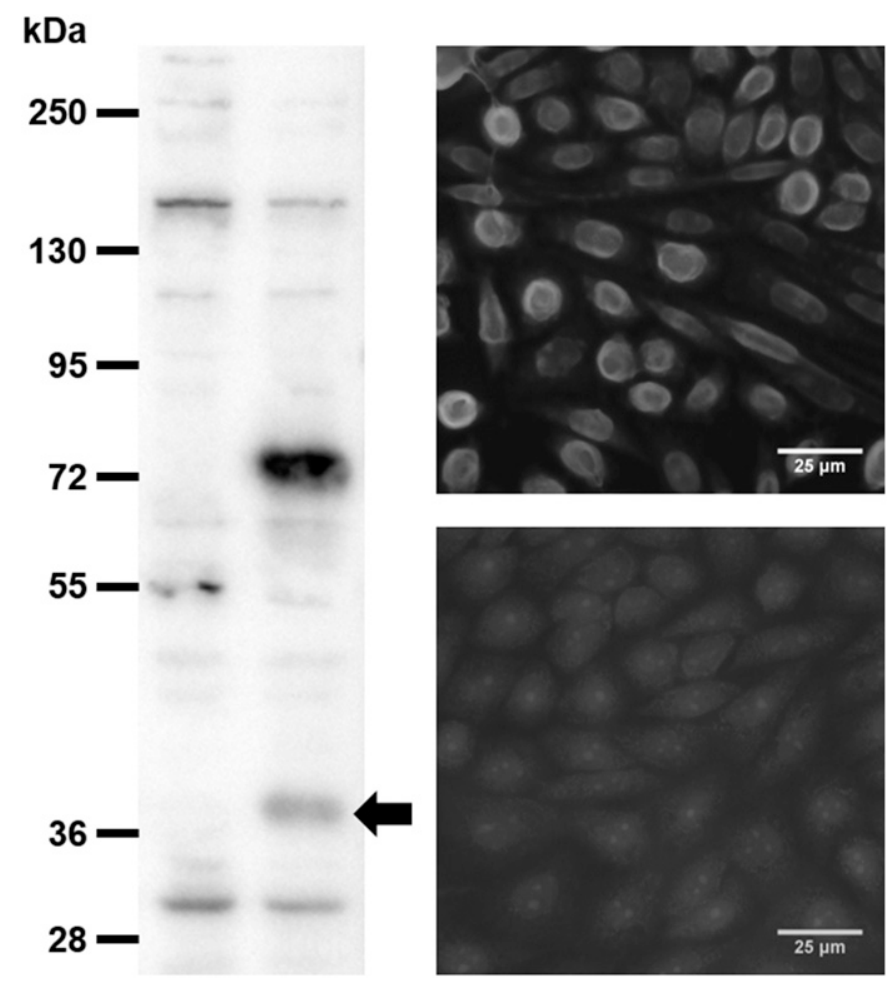

Fig. 2. Continued. 


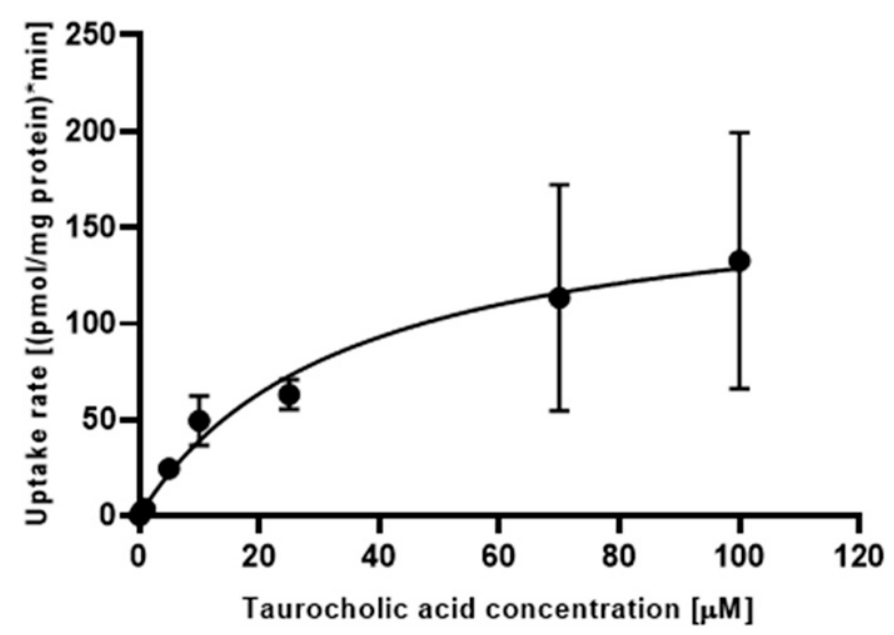

Fig. 3. Kinetic analysis of taurocholate transport into ASBT-CHO cells. Taurocholate uptake was determined as described in Materials and Methods over 1 minute. Uptake was calculated as difference between uptake in the presence of sodium and in the presence of choline. Data are mean \pm S.D. of an experiment with triplicates.

inhibition), all derivatives were weak inhibitors. To exclude an interference of the fluorophores with the transporter, truncated probes AcenNBD, AcenDNS, and AcenPB were also tested but showed no relevant interaction with transport of test substrates (Table 2). The efflux transporter BSEP was strongly inhibited by all BS derivatives, whereas the unconjugated fluorophores showed no interaction (Table 3). In contrast, MRP2 tended to be stimulated rather than inhibited by the NBD and DNS conjugates of the bile acids (Table 3). However, this stimulation was also observed by the respective unconjugated fluorophores AcenNBD and AcenDNS. In contrast, AcenPB showed no interaction with MRP2, whereas CDCenPB stimulated MRP2 transport activity at $10 \mu \mathrm{M}$ but acted as an inhibitor at $100 \mu \mathrm{M}$. The unconjugated AcenPB did not inhibit MRP2mediated transport of estradiol $17 \beta$-D-glucuronide.

Transport of Fluorescent Bile Salt Probes. Finally, we assessed the fluorescent BS derivatives, which were positive in the inhibition assay as potential transport substrates of the investigated organic anion transporters. The data for the uptake transporters are presented in Table 4. Overall, most inhibitory interactions did not result in positive transport data. None of the truncated fluorescent probes turned out to be a substrate of any transporter investigated. As the CenPB

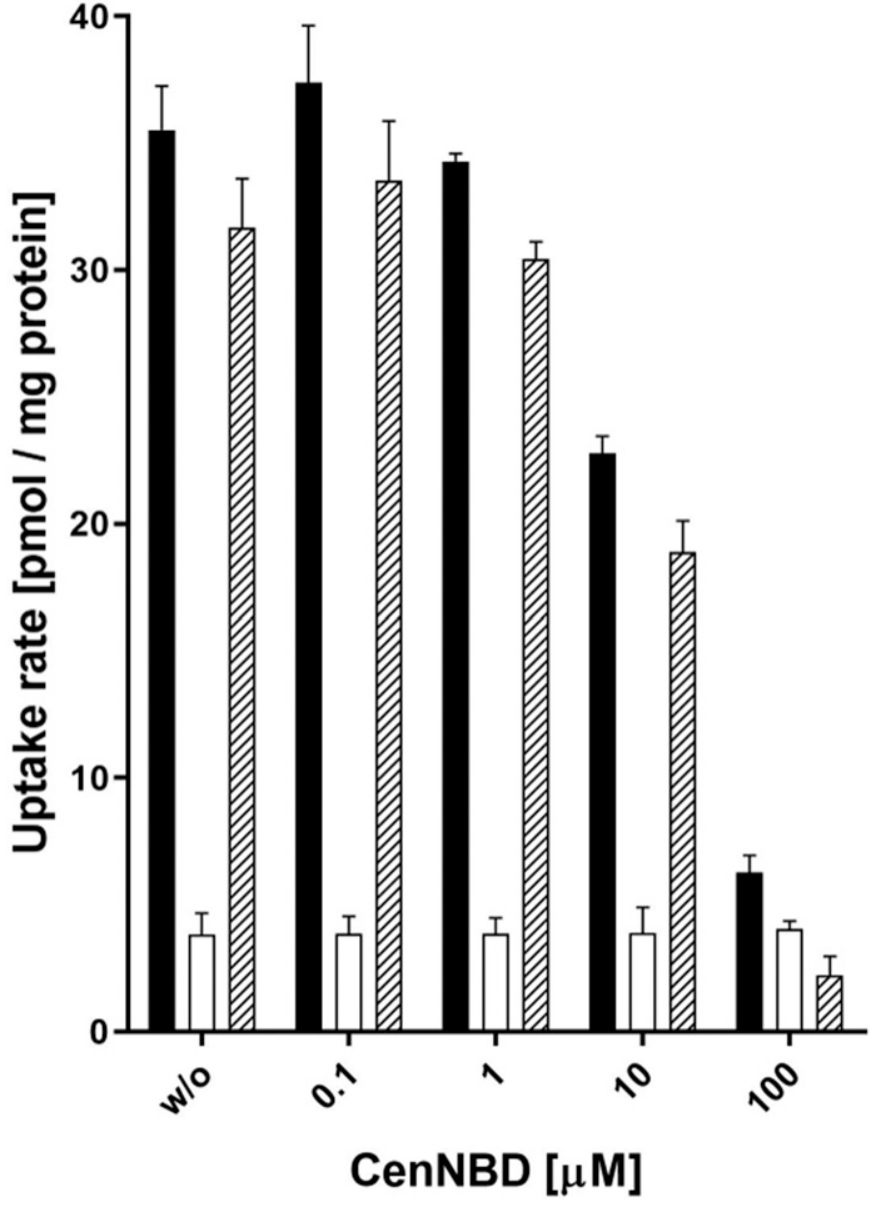

Fig. 4. Inhibition of $1 \mu \mathrm{M}$ taurocholate uptake into WT (open bars) and OATP1B1 CHO cells (closed bars) in the presence of increasing concentrations CenNBD. The difference between OATP1B1 CHO and WT cells (hatched bars) is taken as OATP1B1-mediated transport. Transport was determined for 40 seconds as described in Materials and Methods. Data are mean \pm S.D. of a representative experiment with three technical replicates.

derivatives were neither a substrate for NTCP nor for ASBT, AcenPB was not tested on these two transporters (Table 4). ASBT does not transport any of the tested fluorescent BS derivatives, and NTCP accepts CenDNS as substrate at low capacity. CenNBD is a low-capacity substrate of OATP1B1 and OATP2B1. CenDNS is well transported by OATP1B3 and

TABLE 1

Uptake rates of tested transport systems

Transport experiments were performed as described in Material and Methods using the substrates and conc. given in the table. Uptake was normalized to picomole per milligram protein $\times$ minute. The ratio was calculated by dividing transporter-mediated uptake rates by uptake rates under control conditions. Data are given as mean \pm S.D.

\begin{tabular}{|c|c|c|c|c|c|}
\hline \multirow{2}{*}{ Transport System } & \multirow{2}{*}{ Substrate } & \multicolumn{2}{|c|}{ Conditions } & \multirow{2}{*}{ Ratio } & \multirow{2}{*}{$n$} \\
\hline & & Choline Buffer & Sodium Buffer & & \\
\hline NTCP & $5 \mu \mathrm{M}$ taurocholate & $39.6 \pm 20.9$ & $135 \pm 77$ & $3.6 \pm 1.7$ & 12 \\
\hline ASBT & & WT & Transfected & & \\
\hline OATP1B1 & $1 \mu \mathrm{M}$ estrone-3-sulfate & $6.62 \pm 2.06$ & $\overline{78.8 \pm 30.5}$ & $12.7 \pm 0.9$ & 15 \\
\hline OATP1B3 & $5 \mu \mathrm{M}$ taurocholate & $19.0 \pm 6.9$ & $69.9 \pm 18.9$ & $3.9 \pm 1.1$ & 12 \\
\hline BSEP & $2 \mu \mathrm{M}$ taurocholate & $6.97 \pm 1.77$ & $39.9 \pm 10.8$ & $6.2 \pm 2.8$ & 11 \\
\hline MRP2 & $10 \mu \mathrm{M}$ estradiol $17 \beta$-D-glucuronide & $24.9 \pm 3.2$ & $61.7 \pm 8.3$ & $2.5 \pm 0.4$ & 8 \\
\hline
\end{tabular}




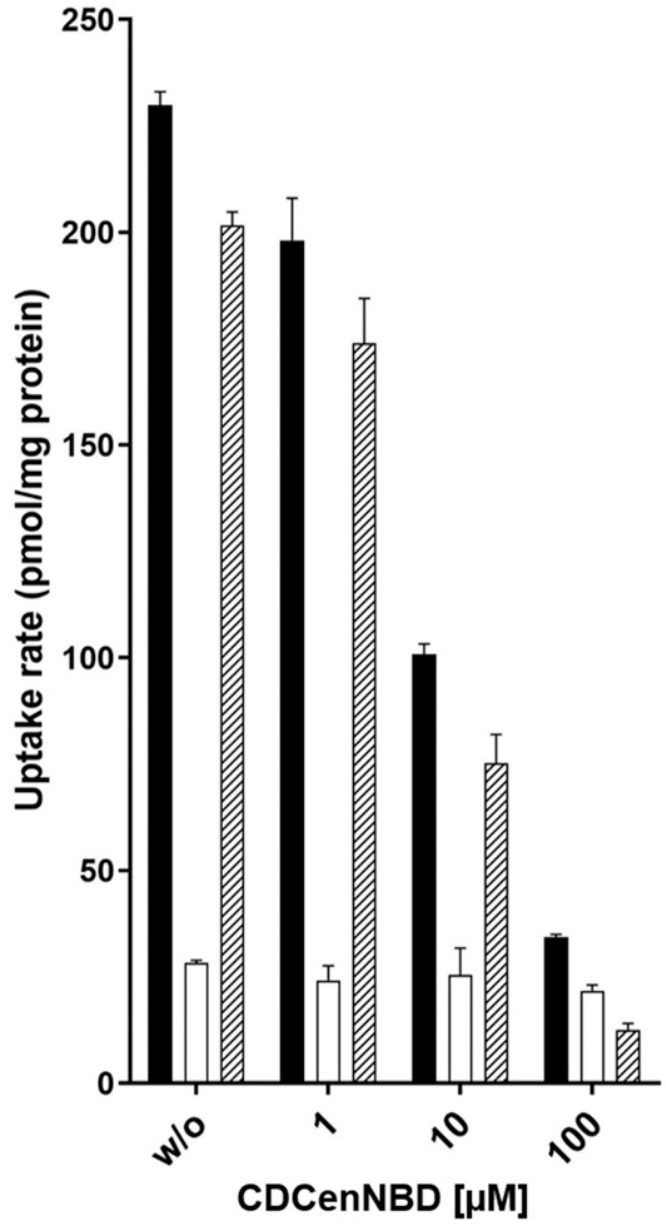

Fig. 5. Inhibition of $2 \mu \mathrm{M}$ taurocholate uptake in the absence (open bars) and presence of ATP (closed bars) into BSEP-expressing insect cell vesicles cells in the presence of increasing concentrations CDCenNBD. The difference between uptake in the presence and absence of ATP (hatched bars) is taken as BSEP-mediated transport. Transport was determined for 5 minutes as described in Materials and Methods. Data are mean \pm S.D. of a representative experiment with three technical replicates.

OATP2B1. Similar to CenNBD, CenPB is a low-capacity substrate of OATP1B1, but it is also a substrate of OATP1B3. CDCenNBD turned out to be a substrate for all hepatocellular OATPs, whereas CDCenDNS and CDCenPB are only transported by OATP1B3 and OATP2B1. Table 5 demonstrates that the ATP-binding cassette $(\mathrm{ABC})$ transporters BSEP and MRP2 do not transport the new fluorescent bile acid derivatives. CenPB (BSEP) and CDCenPB (BSEP and MRP2) were both positive in two independent experiments, however, with large S.D. values. Hence, although these compounds may be used as substrates for BSEP or MRP2, transport experiments will be difficult given the high variability found here, and we would therefore rather conclude that they are not transported.

\section{Discussion}

The aim of the present study was to thoroughly test the impact of BS side-chain modifications with small fluorophores on the interaction with hepatocellular organic anion transporters, both with respect to inhibition and as potential substrates. The main findings are that firstly, all tested uptake
TABLE 2

Inhibition of uptake transporters by fluorescent bile acid derivatives Transport experiments were performed as described in Material and Methods. The following substrates were used: NTCP, OATP1B3: $5 \mu \mathrm{M}$ taurocholate; ASBT: $1 \mu \mathrm{M}$ taurocholate; and OATP1B1 and OATP2B1: $1 \mu \mathrm{M}$ estrone-3-sulfate. Transport was measured for 40 seconds. Substrate uptake values in \% were corrected for uptake into WT CHO cells. Uptake in the absence of inhibitor was set to $100 \%$. Results are the mean from two to four independent experiments with three technical replicates.

\begin{tabular}{|c|c|c|c|c|c|c|}
\hline Compound & [I] & NTCP & OATP1B1 & OATP1B3 & OATP2B1 & ASBT \\
\hline & $\mu M$ & & & & & \\
\hline \multirow[t]{5}{*}{ CenNBD } & 0 & 100 & 100 & 100 & 100 & 100 \\
\hline & 0.1 & 98.5 & 101 & 108 & 90.9 & 95.8 \\
\hline & 1 & 108.3 & 95.1 & 83.0 & 76.6 & 86.5 \\
\hline & 10 & 38.7 & 64.9 & 26.6 & 23.0 & 76.4 \\
\hline & 100 & 4.9 & 9.4 & 22.9 & 3.6 & 64.2 \\
\hline \multirow[t]{5}{*}{ CenDNS } & 0 & 100 & 100 & 100 & 100 & 100 \\
\hline & 0.1 & 85.9 & 98.8 & 82.7 & 94.0 & 85.5 \\
\hline & 1 & 49.1 & 89.2 & 57.0 & 55.5 & 80.1 \\
\hline & 10 & 7.8 & 30.4 & 13.0 & 11.5 & 59.4 \\
\hline & 100 & 2.4 & 16.5 & 41.7 & 7.4 & 47.9 \\
\hline \multirow[t]{5}{*}{ CenPB } & 0 & 100 & 100 & 100 & 100 & 100 \\
\hline & 0.1 & 93.8 & 98.7 & 69.0 & 95.4 & 47.7 \\
\hline & 1 & 73.9 & 93.7 & 62.0 & 89.8 & 40.4 \\
\hline & 10 & 28.9 & 72.7 & 28.6 & 55.0 & 50.4 \\
\hline & 100 & 5.8 & 51.2 & 19.0 & 15.7 & 51.0 \\
\hline \multirow[t]{5}{*}{ CDCenNBD } & 0 & 100 & 100 & 100 & 100 & 100 \\
\hline & 0.1 & 85.6 & 99.7 & 132 & 86.3 & 99.1 \\
\hline & 1 & 67.5 & 95.6 & 95.5 & 85.4 & 108 \\
\hline & 10 & 15.7 & 90.8 & 34.1 & 64.7 & 113 \\
\hline & 100 & 17.7 & 86.8 & 41.2 & 66.9 & 91.7 \\
\hline \multirow{5}{*}{ CDCenDNS } & 0 & 100 & 100 & 100 & 100 & 100 \\
\hline & 0.1 & 92.9 & 99.8 & 83.0 & 87.8 & 68.9 \\
\hline & 1 & 61.8 & 94.3 & 64.0 & 87.9 & 123 \\
\hline & 10 & 46.6 & 83.3 & 55.9 & 73.0 & 164 \\
\hline & 100 & 46.2 & 84.0 & 50.5 & 62.7 & 109 \\
\hline \multirow[t]{5}{*}{ CDCenPB } & 0 & 100 & 100 & 100 & 100 & 100 \\
\hline & 0.1 & 64.5 & 91.9 & 56.7 & 98.0 & 43.4 \\
\hline & 1 & 29.6 & 71.5 & 37.5 & 60.5 & 42.3 \\
\hline & 10 & 6.7 & 36.3 & 28.8 & 13.2 & 48.6 \\
\hline & 100 & 3.9 & 3.1 & 19.8 & 3.7 & 45.3 \\
\hline \multirow[t]{5}{*}{ AcenNBD } & 0 & 100 & 100 & 100 & 100 & 100 \\
\hline & 0.1 & 75.8 & 93.2 & 87.5 & 83.0 & 79.0 \\
\hline & 1 & 87.6 & 89.4 & 103 & 86.7 & 98.8 \\
\hline & 10 & 118 & 86.3 & 103 & 83.7 & 100 \\
\hline & 100 & 75.8 & 78.3 & 88.4 & 49.1 & 144 \\
\hline \multirow[t]{5}{*}{ AcenDNS } & 0 & 100 & 100 & 100 & 100 & 100 \\
\hline & 0.1 & 92.7 & 99.6 & 109 & 95.8 & 82.0 \\
\hline & 1 & 110 & 94.8 & 102 & 95.7 & 92.2 \\
\hline & 10 & 120 & 93.5 & 94.8 & 114 & 99.6 \\
\hline & 100 & 80.2 & 90.8 & 69.9 & 147 & 101 \\
\hline \multirow{5}{*}{ AcenPB } & 0 & ND & 100 & 100 & 100 & ND \\
\hline & 0.1 & ND & 99.7 & 81.3 & 99.2 & ND \\
\hline & 1 & ND & 96.8 & 63.8 & 96.4 & ND \\
\hline & 10 & ND & 96.1 & 62.5 & 94.3 & ND \\
\hline & 100 & ND & 89.0 & 62.5 & 68.8 & ND \\
\hline
\end{tabular}

$\mathrm{ND}$, not determined

transporters are inhibited by at least one novel bile acid derivative (Table 2), whereby the interaction with ASBT was more restricted than interaction with other transporters. Secondly, neither NTCP (with the exception of a low transport activity for CenDNS) nor ASBT transport fluorescent BS derivatives, whereas all investigated OATPs accept more than one derivative (Table 4). Thirdly, the efflux transporter BSEP is subject to inhibition by all fluorescent bile acid derivatives, whereas MRP2 is not inhibited but rather stimulated in some instances (Table 3). Fourthly, efflux transport activity of the new BS derivatives is, as compared with the uptake transporters, very restricted (Table 5).

Given that, in particular, OATPs (Stieger and Hagenbuch, 2014) as well as MRP2 (van der Schoor et al., 2015) have a very broad substrate specificity and both are able transporting fluorescent dyes (Cantz et al., 2000; Brouwer et al., 2013; 
TABLE 3

Inhibition of efflux transporters by fluorescent bile acid derivatives Transport experiments were performed as described in Material and Methods. The following substrates were used: BSEP $2 \mu \mathrm{M}$ taurocholate and MRP2 $10 \mu \mathrm{M}$ estradiol $17 \beta$-D-glucuronide. Transport was measured for 5 minutes. Substrate uptake values in \% were corrected for transport in the absence of ATP, and uptake in the absence of inhibitor was set to $100 \%$. Results are the mean from two to four independent experiments with three technical replicates.

\begin{tabular}{|c|c|c|c|}
\hline Compound & [I] & BSEP & MRP2 \\
\hline & $\mu M$ & & \\
\hline \multirow{4}{*}{ CenNBD } & 0 & 100 & 100 \\
\hline & 1 & 87.5 & 94.5 \\
\hline & 10 & 46.0 & 124 \\
\hline & 100 & 12.0 & 140 \\
\hline \multirow[t]{4}{*}{ CenDNS } & 0 & 100 & 100 \\
\hline & 1 & 82.0 & 78.5 \\
\hline & 10 & 50.5 & 106 \\
\hline & 100 & 6.0 & 152 \\
\hline \multirow[t]{4}{*}{ CenPB } & 0 & 100 & 100 \\
\hline & 1 & 80.0 & 94.5 \\
\hline & 10 & 58.0 & 94.5 \\
\hline & 100 & 16.5 & 91.0 \\
\hline \multirow{4}{*}{ CDCenNBD } & 0 & 100 & 100 \\
\hline & 1 & 67.5 & 122 \\
\hline & 10 & 40.5 & 151 \\
\hline & 100 & 6.5 & 228 \\
\hline \multirow[t]{4}{*}{ CDCenDNS } & 0 & 100 & 100 \\
\hline & 1 & 85.5 & 115 \\
\hline & 10 & 43.0 & 132 \\
\hline & 100 & 25.5 & 179 \\
\hline \multirow[t]{4}{*}{ CDCenPB } & 0 & 100 & 100 \\
\hline & 1 & 61.5 & 107 \\
\hline & 10 & 13.0 & 151 \\
\hline & 100 & 0.5 & 59.3 \\
\hline \multirow[t]{4}{*}{ AcenNBD } & 0 & 100 & 100 \\
\hline & 1 & 103 & 136 \\
\hline & 10 & 97.5 & 132 \\
\hline & 100 & 94.5 & 145 \\
\hline \multirow[t]{4}{*}{ AcenDNS } & 0 & 100 & 100 \\
\hline & 1 & 96.5 & 139 \\
\hline & 10 & 97.0 & 136 \\
\hline & 100 & 87.5 & 151 \\
\hline \multirow{4}{*}{ AcenPB } & 0 & 100 & 100 \\
\hline & 1 & 116 & 99 \\
\hline & 10 & 108 & 95 \\
\hline & 100 & 102 & 93 \\
\hline
\end{tabular}

Patik et al., 2015), special care was taken to separate the desired reaction products (fluorescent bile acid conjugates) with great purity from the fluorescent dye starting materials after the synthetic coupling reactions. As an additional control, possible interactions of the fluorescent dyes with the investigated transporters were probed. To this end, bile acid-truncated fluorescent probes AcenNBD, AcenDNS, and AcenPB were tested as potential inhibitors and substrates.
Not one of these fluorophores was found to be a substrate. Using the molecules as inhibitors, AcenNB weakly interacted with OATP2B1, whereas AcenPB and AcenDNS weakly inhibited OATP1B3. This interaction is not surprising, as different fluorescent molecules have been shown to be transported by OATPs (Patik et al., 2015, 2018). In contrast, AcenDNS exhibited stimulation of OATP2B1 activity at high concentration. A stimulation of OATP2B1 transport activity by small molecules has been reported for prostaglandins (Pizzagalli et al., 2003) or steroids (Grube et al., 2006), but such stimulation is not restricted to OATP2B1 (Stieger and Hagenbuch, 2014). All fluorescent bile acid derivatives interacted with the tested transporters. As all of these uptake transporters are bile salt and bile acid transporters, we conclude that coupling of fluorophores to the side chain does not prevent the final probes from interacting with the respective bile acid binding sites on the transporters. Notably, CDCenDNS tended to transiently stimulate the transport activity of ASBT. This, to our knowledge, is a new finding, and a similar observation was made for silibinin for the lowaffinity binding site of OATP1B1 (Wlcek et al., 2013). The truncated fluorescent probes did not interact with BSEP, but AcenNBD and AcenDNS were able to stimulate the transport activity of MRP2. A stimulation of the transport activity of MRP2 has also been reported for bosentan (Mano et al., 2007) and other selected drugs (Lagas et al., 2009; Gilibili et al., 2018).

In essence, neither NTCP nor ASBT transported any of the tested substrates. However, it cannot be concluded from this finding that modification of the side chain prevents bile salts to be substrates of NTCP. Since endogenous conjugation of taurine as well as glycine to the side chain of bile acids is via amide bond, we also coupled the fluorescent dyes to the side chain by an amide bond in the present work, similarly to an earlier study (Maglova et al., 1995). These authors report that two different side-chain derivatives of cholate were taken up in part in a sodium-dependent manner into primary rat hepatocytes, whereas an identical side-chain derivative of chenodeoxycholate displayed no dependence on sodium (Maglova et al., 1995). A fluorescein side-chain derivative of glycocholate was shown to be imported into rat hepatocytes in a sodium-dependent manner, indicating transport by rat NTCP (Grüne et al., 1996). On the other hand, a lysyl-fluorescein side-chain derivative of cholate was not transported by NTCP (de Waart et al., 2010). A fluorescent side-chain derivative of cholate has previously been shown to be transported by

TABLE 4

Transport of fluorescent bile acid derivatives by uptake transporters

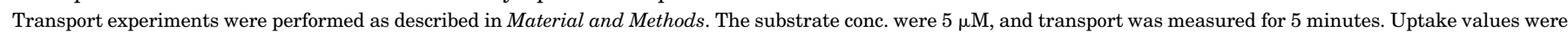

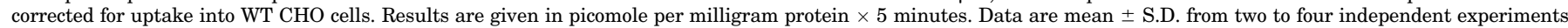
with three technical replicates.

\begin{tabular}{|c|c|c|c|c|c|}
\hline Compound & NTCP & OATP1B1 & OATP1B3 & OATP2B1 & ASBT \\
\hline CenNBD & NT & $67.0 \pm 30.0$ & NT & $26.1 \pm 13.7$ & NT \\
\hline CenDNS & $20.3 \pm 10.0$ & NT & $1577 \pm 509$ & $175 \pm 20.2$ & NT \\
\hline CenPB & NT & $21.6 \pm 13.1$ & $306 \pm 188$ & NT & NT \\
\hline CDCenNBD & NT & $908 \pm 309$ & $8545 \pm 3200$ & $1779 \pm 405$ & NT \\
\hline CDCenDNS & NT & NT & $1524 \pm 311$ & $141 \pm 26.61$ & NT \\
\hline CDCenPB & NT & NT & $162 \pm 59.7$ & $60.5 \pm 18.4$ & NT \\
\hline AcenNBD & NT & NT & NT & NT & NT \\
\hline AcenDNS & NT & NT & NT & NT & NT \\
\hline AcenPB & ND & NT & NT & NT & ND \\
\hline
\end{tabular}

ND, not determined; NT, no transport. 


\section{TABLE 5}

Transport of fluorescent bile acid derivatives by efflux transporters Transport experiments were performed as described in Material and Methods. The substrate conc. were $5 \mu \mathrm{M}$, and transport was measured for 5 minutes. Uptake values were corrected for uptake in the absence of ATP. Results are given in picomole per milligram protein $\times 5$ minutes. Data are mean \pm S.D. from two to four independent experiments with three technical replicates.

\begin{tabular}{lcc}
\hline Compound & BSEP & MRP2 \\
\hline CenNBD & $\mathrm{NT}$ & $\mathrm{NT}$ \\
CenDNS & $\mathrm{NT}$ & $\mathrm{NT}$ \\
CenPB & $81.4 \pm 75.1$ & $\mathrm{NT}$ \\
CDCenNBD & $\mathrm{NT}$ & $\mathrm{NT}$ \\
CDCenDNS & $\mathrm{NT}$ & $\mathrm{NT}$ \\
CDCenPB & $115 \pm 103$ & $404 \pm 355$ \\
AcenNBD & $\mathrm{NT}$ & $\mathrm{NT}$ \\
AcenDNS & $\mathrm{NT}$ & $\mathrm{NT}$ \\
AcenPB & $\mathrm{NT}$ & $\mathrm{NT}$ \\
\hline
\end{tabular}

NT, no transport.

heterologously expressed NTCP and rNTCP (De Bruyn et al., 2014). While developing fluorinated BS derivatives amenable to positron emission tomography studies, it was observed that a lithocholic acid derivative with a modified side chain was accepted by NTCP as substrate (Testa et al., 2017). Hence, based on the current literature, it is hard to predict the impact of side-chain modification of bile acids affecting their substrate properties for NTCP. It should be pointed out, however, that the substrate pattern of NTCP not only includes bile salts but also other organic anions as well as some drugs (Anwer and Stieger, 2014). It is interesting to note that a substrate pharmacophore for NTCP without consideration of the side chain has been presented (Greupink et al., 2012; Dong et al., 2015). There is a consensus that the substrate specificity of ASBT is much narrower than that of NTCP (Claro da Silva et al., 2013; Anwer and Stieger, 2014; Dawson and Karpen, 2015). In this context, the results for ASBT were not surprising.

Given the broad substrate specificity of OATPs, we expected that they would also transport some of our novel BS derivatives (Table 4). Here, it is interesting to note that OATP2B1 transports five out of the six tested fluorescent BS probes. This is in contrast with an earlier study, in which we found no transport of conjugated bile acids by OATP2B1 (Kullak-Ublick et al., 2001). It is, however, very reassuring that none of the fluorescent dyes we used was a substrate of the tested OATPs. Hence, these dyes might be coupled to different positions of the bile acid skeleton (Yamaguchi et al., 2006).

Given that a fluorescent side-chain derivative of bile salts was reported to be a BSEP substrate (Yamaguchi et al., 2010), it was somewhat unexpected that none of our derivatives was transported by BSEP. However, our data fit well with the concept that BSEP transports almost exclusively conjugated bile acids (Stieger, 2011). Although all derivatives were inhibitors of BSEP, none of the truncated fluorescent probes significantly interacted with this transporter. Although MRP2 transports a wide variety of endogenous metabolites as well as drug and xenobiotic metabolites (Keppler, 2011), none of our bile acid derivatives was a good substrate. This is interesting in view of the fact that some of our derivatives were able to stimulate transport activity of a prototypical substrate of MRP2. This may, at least in part, be explained by the rather complex transport kinetics of MRP2 (Guyot et al., 2014). Again, none of the fluorescent dyes used here was transported by MRP2. However, it should be kept in mind that extrapolation of driving forces from in vitro systems to in vivo situations is very difficult at best. It can therefore not be excluded that in an in vivo model CDCenPB might be taken up into a liver system and secreted into bile. As it is possible to keep livers with continuous perfusion alive out of bodies for up to a week now (Eshmuminov et al., 2020, 2021), CDCenPB for instance could be evaluated as a dynamic liver function marker in ex situ perfused (human) livers.

In conclusion, side-chain modification of bile acids does not prevent the conjugates interacting with organic anion and bile salt uptake and efflux transporters. However, the new fluorescent compounds are not transport substrates for bile acid uptake transporters NCTP and ASBT. Furthermore, with the exception of CDCenPB, they are not transported by the canalicular efflux transporters and thus are not suitable for in vivo studies. Nevertheless, these new fluorescent BS derivatives have some potential as imaging agents if substrate uptake into hepatocytes or cells is to be followed. Since CDCenPB may be both a substrate of the uptake transporters OATP1B3 and OATP2B1 as well as of the canalicular efflux transporters BSEP and MRP2, future studies in in vivo models of bile formation with this compound are warranted.

\section{Acknowledgments}

We thank the analytical services from the Department of Chemistry and Biochemistry, University of Bern, Switzerland for measuring NMR and MS spectra of synthetic intermediates and final compounds.

\section{Authorship Contributions}

Participated in research design: Leuenberger, Häusler, Stieger, Lochner.

Conducted experiments: Leuenberger, Häusler, Höhn, Euler.

Contributed new reagents or analytic tools: Leuenberger.

Performed data analysis: Leuenberger, Häusler, Stieger, Lochner.

Wrote or contributed to the writing of the manuscript: Leuenberger, Stieger, Lochner.

\section{References}

Aithal GP, Watkins PB, Andrade RJ, Larrey D, Molokhia M, Takikawa H, Hunt CM, Wilke RA, Avigan M, Kaplowitz N, et al. (2011) Case definition and phenotype standardization in drug-induced liver injury. Clin Pharmacol Ther 89:806-815.

Anwer MS and Stieger B (2014) Sodium-dependent bile salt transporters of the SLC10A transporter family: more than solute transporters. Pflugers Arch 466: $77-89$

Brouwer KL, Keppler D, Hoffmaster KA, Bow DA, Cheng Y, Lai Y, Palm JE, Stieger B, and Evers R; International Transporter Consortium (2013) In vitro methods to support transporter evaluation in drug discovery and development [published correction appears in Clin Pharmacol Ther (2013) 94:412]. Clin Pharmacol Ther 94: 95-112.

Bull LN and Thompson RJ (2018) Progressive familial intrahepatic cholestasis. Clin Liver Dis 22:657-669.

Cantz T, Nies AT, Brom M, Hofmann AF, and Keppler D (2000) MRP2, a human conjugate export pump, is present and transports fluo 3 into apical vacuoles of Hep G2 cells. Am J Physiol Gastrointest Liver Physiol 278:G522-G531.

Cieslak KP, Baur O, Verheij J, Bennink RJ, and van Gulik TM (2016) Liver function declines with increased age. HPB (Oxford) 18:691-696.

Claro da Silva T, Polli JE, and Swaan PW (2013) The solute carrier family 10 (SLC10): beyond bile acid transport. Mol Aspects Med 34:252-269.

Corsini A and Bortolini M (2013) Drug-induced liver injury: the role of drug metabolism and transport. J Clin Pharmacol 53:463-474.

Craddock AL, Love MW, Daniel RW, Kirby LC, Walters HC, Wong MH, and Dawson PA (1998) Expression and transport properties of the human ileal and renal sodium-dependent bile acid transporter. Am J Physiol 274:G157-G169.

Dawson PA and Karpen SJ (2015) Intestinal transport and metabolism of bile acids. $J$ Lipid Res 56:1085-1099.

De Bruyn T, Fattah S, Stieger B, Augustijns P, and Annaert P (2011) Sodium fluorescein is a probe substrate for hepatic drug transport mediated by OATP1B1 and OATP1B3. J Pharm Sci 100:5018-5030.

De Bruyn T, Sempels W, Snoeys J, Holmstock N, Chatterjee S, Stieger B, Augustijns P, Hofkens J, Mizuno H, and Annaert P (2014) Confocal imaging with a fluorescent bile acid analogue closely mimicking hepatic taurocholate disposition. J Pharm Sci 103:1872-1881.

de Waart DR, Häusler S, Vlaming ML, Kunne C, Hänggi E, Gruss HJ, Oude Elferink RP, and Stieger B (2010) Hepatic transport mechanisms of cholyl-L-lysyl-fluorescein. $J$ Pharmacol Exp Ther 334:78-86. 
de Waart DR, Paulusma CC, Kunne C, and Oude Elferink RP (2006) Multidrug resistance associated protein 2 mediates transport of prostaglandin E2. Liver Int 26:362-368.

Dong Z, Ekins S, and Polli JE (2015) A substrate pharmacophore for the human sodium taurocholate co-transporting polypeptide. Int J Pharm 478:88-95.

Eshmuminov D, Becker D, Bautista Borrego L, Hefti M, Schuler MJ, Hagedorn C, Muller X, Mueller M, Onder C, Graf R, et al. (2020) An integrated perfusion machine preserves injured human livers for 1 week. Nat Biotechnol 38:189-198.

Eshmuminov D, Schuler MJ, Becker D, Bautista Borrego L, Mueller M, Hagedorn C, Häusler S, Steiger J, Tibbitt MW, Dutkowski P, et al. (2021) Bile formation in longterm ex situ perfused livers. Surgery 169:894-902 DOI: $10.1016 /$ j.surg.2020.11.042.

Esteller A (2008) Physiology of bile secretion. World J Gastroenterol 14:5641-5649.

Frosch B and Wagener H (1967) Quantitative determination of conjugated bile acids in serum in acute hepatitis. Nature 213:404-405.

Gerloff T, Stieger B, Hagenbuch B, Madon J, Landmann L, Roth J, Hofmann AF, and Meier PJ (1998) The sister of P-glycoprotein represents the canalicular bile salt export pump of mammalian liver. J Biol Chem 273:10046-10050.

Gilibili RR, Kurawattimath V, Murali BV, Lai Y, Mariappan TT, Shen H, and Chatterjee $S$ (2018) In vitro stimulation of multidrug resistance-associated protein 2 function is not reproduced in vivo in rats. Pharmaceutics 10:125.

Greupink R, Nabuurs SB, Zarzycka B, Verweij V, Monshouwer M, Huisman MT, and Russel FG (2012) In silico identification of potential cholestasis-inducing agents via modeling of $\mathrm{Na}(+)$-dependent taurocholate cotransporting polypeptide substrate specificity. Toxicol Sci 129:35-48.

Grube M, Köck K, Karner S, Reuther S, Ritter CA, Jedlitschky G, and Kroemer HK (2006) Modification of OATP2B1-mediated transport by steroid hormones. Mol Pharmacol 70:1735-1741.

Grüne S, Meng XJ, and Weinman SA (1996) cAMP stimulates fluorescent bile acid uptake into hepatocytes by membrane hyperpolarization. Am J Physiol 270: G339-G346.

Gui C, Miao Y, Thompson L, Wahlgren B, Mock M, Stieger B, and Hagenbuch B (2008) Effect of pregnane X receptor ligands on transport mediated by human OATP1B1 and OATP1B3. Eur J Pharmacol 584:57-65.

Guyot C, Hofstetter L, and Stieger B (2014) Differential effects of membrane cholesterol content on the transport activity of multidrug resistance-associated protein 2 (ABCC2) and of the bile salt export pump (ABCB11). Mol Pharmacol 85:909-920.

Hagenbuch B and Stieger B (2013) The SLCO (former SLC21) superfamily of transporters. Mol Aspects Med 34:396-412.

Halilbasic E, Claudel T, and Trauner M (2013) Bile acid transporters and regulatory nuclear receptors in the liver and beyond. J Hepatol 58:155-168.

Hoekstra LT, de Graaf W, Nibourg GAA, Heger M, Bennink RJ, Stieger B, and van Gulik TM (2013) Physiological and biochemical basis of clinical liver function tests: a review. Ann Surg 257:27-36.

Holzinger F, Schteingart CD, Ton-Nu H-T, Cerrè C, Steinbach JH, Yeh H-Z and Hofmann AF (1998) Transport of fluorescent bile acids by the isolated perfused rat liver: kinetics, sequestration, and mobilization. Hepatology 28:510-520.

Holzinger F, Schteingart CD, Ton-Nu HT, Eming SA, Monte MJ, Hagey LR, and Hofmann AF (1997) Fluorescent bile acid derivatives: relationship between chemical structure and hepatic and intestinal transport in the rat. Hepatology $\mathbf{2 6}$ : 1263-1271.

Huber RD, Gao B, Sidler Pfändler MA, Zhang-Fu W, Leuthold S, Hagenbuch B Folkers G, Meier PJ, and Stieger B (2007) Characterization of two splice variants of human organic anion transporting polypeptide $3 \mathrm{~A} 1$ isolated from human brain. Am $J$ Physiol Cell Physiol 292:C795-C806.

Keppler D (2011) Multidrug resistance proteins (MRPs, ABCCs): importance for pathophysiology and drug therapy. Handb Exp Pharmacol 201:299-323 Available from: $10.1007 / 978-3-642-14541-48$

Kjærgaard K, Frisch K, Sørensen M, Munk OL, Hofmann AF, Horsager J, Schacht AC, Erickson M, Shapiro D, and Keiding S (2021) Obeticholic acid improves hepatic bile acid excretion in patients with primary biliary cholangitis. $J$ Hepatol 74:58-65

Kullak-Ublick GA, Ismair MG, Stieger B, Landmann L, Huber R, Pizzagalli F, Fattinger K, Meier PJ, and Hagenbuch B (2001) Organic anion-transporting polypeptide B (OATP-B) and its functional comparison with three other OATPs of human liver. Gastroenterology 120:525-533.

Lagas JS, van der Kruijssen CM, van de Wetering K, Beijnen JH, and Schinkel AH (2009) Transport of diclofenac by breast cancer resistance protein (ABCG2) and stimulation of multidrug resistance protein 2 (ABCC2)-mediated drug transport by diclofenac and benzbromarone. Drug Metab Dispos 37:129-136.

LaRusso NF, Hoffman NE, Hofmann AF, and Korman MG (1975) Validity and sensitivity of an intravenous bile acid tolerance test in patients with liver disease. $N$ Engl J Med 292:1209-1214.

Maglova LM, Jackson AM, Meng XJ, Carruth MW, Schteingart CD, Ton-Nu HT, Hofmann AF, and Weinman SA (1995) Transport characteristics of three fluorescent conjugated bile acid analogs in isolated rat hepatocytes and couplets. Hepatology 22:637-647.

Mahdi ZM, Synal-Hermanns U, Yoker A, Locher KP, and Stieger B (2016) Role of multidrug resistance protein 3 in antifungal-induced cholestasis. Mol Pharmacol 90:23-34.

Mano Y, Usui T, and Kamimura H (2007) Substrate-dependent modulation of UDPglucuronosyltransferase 1A1 (UGT1A1) by propofol in recombinant human UGT1A1 and human liver microsomes. Basic Clin Pharmacol Toxicol 101:211-214.
Milkiewicz P, Saksena S, Cardenas T, Mills CO, and Elias E (2000) Plasma elimination of cholyl-lysyl-fluorescein $(\mathrm{CLF})$ : a pilot study in patients with liver cirrhosis. Liver 20:330-334.

Noé J, Stieger B, and Meier PJ (2002) Functional expression of the canalicular bile salt export pump of human liver. Gastroenterology 123:1659-1666.

Obaidat A, Roth M, and Hagenbuch B (2012) The expression and function of organic anion transporting polypeptides in normal tissues and in cancer. Annu Rev Pharmacol Toxicol 52:135-151.

Palermo DP, DeGraaf ME, Marotti KR, Rehberg E, and Post LE (1991) Production of analytical quantities of recombinant proteins in Chinese hamster ovary cells using sodium butyrate to elevate gene expression. J Biotechnol 19:35-47.

Patik I, Kovacsics D, Német O, Gera M, Várady G, Stieger B, Hagenbuch B, Szakács G, and Özvegy-Laczka C (2015) Functional expression of the 11 human Organic Anion Transporting Polypeptides in insect cells reveals that sodium fluorescein is a general OATP substrate. Biochem Pharmacol 98:649-658.

Patik I, Székely V, Német O, Szepesi Á, Kucsma N, Várady G, Szakács G, Bakos É, and Özvegy-Laczka C (2018) Identification of novel cell-impermeant fluorescent substrates for testing the function and drug interaction of Organic AnionTransporting Polypeptides, OATP1B1/1B3 and 2B1. Sci Rep 8:2630.

Paumgartner G, Vasella DL, Herz R, Reichen J, and Preisig R (1979) [Hepatic extraction of taurocholate and indocyanine green in patients with liver disease (author's transl)]. Z Gastroenterol 17:753-761.

Pizzagalli F, Varga Z, Huber RD, Folkers G, Meier PJ, and St-Pierre MV (2003) Identification of steroid sulfate transport processes in the human mammary gland. $J$ Clin Endocrinol Metab 88:3902-3912.

Pollheimer MJ, Fickert P, and Stieger B (2014) Chronic cholestatic liver diseases: clues from histopathology for pathogenesis. Mol Aspects Med 37:35-56.

Rais R, Acharya C, Mackerell AD, and Polli JE (2010) Structural determinants for transport across the intestinal bile acid transporter using $\mathrm{C}-24$ bile acid conjugates. Mol Pharm 7:2240-2254.

Sakka SG (2018) Assessment of liver perfusion and function by indocyanine green in the perioperative setting and in critically ill patients. $J$ Clin Monit Comput 32: 787-796.

Selvapatt N, Singanayagam A, Wendon J, and Antoniades CG (2014) Understanding infection susceptibility in patients with acute-on-chronic liver failure. Intensive Care Med 40:1363-1366.

Smith PK, Krohn RI, Hermanson GT, Mallia AK, Gartner FH, Provenzano MD, Fujimoto EK, Goeke NM, Olson BJ, and Klenk DC (1985) Measurement of protein using bicinchoninic acid. Anal Biochem 150:76-85.

Stieger B (2011) The role of the sodium-taurocholate cotransporting polypeptide (NTCP) and of the bile salt export pump (BSEP) in physiology and pathophysiology of bile formation. Handb Exp Pharmacol 201:205-259.

Stieger B and Hagenbuch B (2014) Organic anion-transporting polypeptides. Curr Top Membr 73:205-232.

Stieger B, Heger M, de Graaf W, Paumgartner G, and van Gulik T (2012) The emerging role of transport systems in liver function tests. Eur $J$ Pharmacol 675:1-5.

Testa A, Dall'Angelo S, Mingarelli M, Augello A, Schweiger L, Welch A, Elmore CS, Sharma P, and Zanda M (2017) Design, synthesis, in vitro characterization and preliminary imaging studies on fluorinated bile acid derivatives as PET tracers to study hepatic transporters. Bioorg Med Chem 25:963-976.

Treiber A, Schneiter R, Häusler S, and Stieger B (2007) Bosentan is a substrate of human OATP1B1 and OATP1B3: inhibition of hepatic uptake as the common mechanism of its interactions with cyclosporin A, rifampicin, and sildenafil. Drug Metab Dispos 35:1400-1407.

van der Schoor LW, Verkade HJ, Kuipers F, and Jonker JW (2015) New insights in the biology of ABC transporters ABCC2 and ABCC3: impact on drug disposition. Expert Opin Drug Metab Toxicol 11:273-293.

Wlcek K, Koller F, Ferenci P, and Stieger B (2013) Hepatocellular organic aniontransporting polypeptides (OATPs) and multidrug resistance-associated protein 2 (MRP2) are inhibited by silibinin. Drug Metab Dispos 41:1522-1528.

Yamaguchi H, Okada M, Akitaya S, Ohara H, Mikkaichi T, Ishikawa H, Sato M, Matsuura M, Saga T, Unno M, et al. (2006) Transport of fluorescent chenodeoxycholic acid via the human organic anion transporters OATP1B1 and OATP1B3. J Lipid Res 47:1196-1202.

Yamaguchi K, Murai T, Yabuuchi H, Hui SP, and Kurosawa T (2010) Measurement of bile salt export pump transport activities using a fluorescent bile acid derivative. Drug Metab Pharmacokinet 25:214-219.

Zhao W, Zitzow JD, Ehresman DJ, Chang SC, Butenhoff JL, Forster J, and Hagenbuch B (2015) Na+/taurocholate cotransporting polypeptide and apical sodium-dependent bile acid transporter are involved in the disposition of perfluoroalkyl sulfonates in humans and rats. Toxicol Sci 146:363-373.

Address correspondence to: Martin Lochner, Institute of Biochemistry and Molecular Medicine, University of Bern, Bühlstrasse 28, 3012 Bern, Switzerland. E-mail: martin.lochner@ibmm.unibe.ch; or Bruno Stieger, Department of Clinical Pharmacology and Toxicology, University Hospital Zürich, Rämistrasse 100, 8091 Zürich, Switzerland. E-mail: bruno.stieger@uzh.ch 